\title{
Ribosome heterogeneity in Drosophila melanogaster gonads through paralog-switching
}

\author{
Tayah Hopes ${ }^{1,2, \uparrow}$, Karl Norris ${ }^{1,2,3, \uparrow}$, Michaela Agapiou ${ }^{1,2,3}$, Charley G. P. McCarthy ${ }^{4}$, \\ Philip A. Lewis ${ }^{5}$, Mary J. O'Connell ${ }^{4}$, Juan Fontana ${ }^{1,3,{ }^{*}}$ and Julie L. Aspden ${ }^{\oplus 1,2,{ }^{*}}$ \\ ${ }^{1}$ School of Molecular and Cellular Biology, Faculty of Biological Sciences, University of Leeds, Leeds LS2 9JT, UK, \\ ${ }^{2}$ LeedsOmics, University of Leeds, Leeds, UK, ${ }^{3}$ Astbury Centre for Structural Molecular Biology, University of Leeds, \\ Leeds LS2 9JT, UK, ${ }^{4}$ School of Life Sciences, Faculty of Medicine and Health Sciences, The University of \\ Nottingham, Nottingham NG7 2RD, UK and ${ }^{5}$ School of Cellular and Molecular Medicine, Faculty of Life Sciences, \\ University of Bristol, Bristol BS8 1TD, UK
}

Received May 28, 2021; Revised June 23, 2021; Editorial Decision June 24, 2021; Accepted July 02, 2021

\begin{abstract}
Ribosomes have long been thought of as homogeneous macromolecular machines, but recent evidence suggests they are heterogeneous and could be specialised to regulate translation. Here, we have characterised ribosomal protein heterogeneity across 4 tissues of Drosophila melanogaster. We find that testes and ovaries contain the most heterogeneous ribosome populations, which occurs through a combination of paralog-enrichment and paralogswitching. We have solved structures of ribosomes purified from in vivo tissues by cryo-EM, revealing differences in precise ribosomal arrangement for testis and ovary $80 \mathrm{~S}$ ribosomes. Differences in the amino acid composition of paralog pairs and their localisation on the ribosome exterior indicate paralogswitching could alter the ribosome surface, enabling different proteins to regulate translation. One testisspecific paralog-switching pair is also found in humans, suggesting this is a conserved site of ribosome heterogeneity. Overall, this work allows us to propose that mRNA translation might be regulated in the gonads through ribosome heterogeneity, providing a potential means of ribosome specialisation.
\end{abstract}

\section{INTRODUCTION}

Protein synthesis is essential across the tree of life and undertaken by the highly conserved macromolecular complex of 'the ribosome'. mRNA translation is regulated at many levels, but until recently the ribosome itself was not thought to be part of this control system. Recent studies have suggested that ribosomes can contribute to gene expression regulation, through specific changes in their composition, i.e. specialization (1-3). These 'specialised ribosomes' are thought to contribute to the translation of specific mRNA pools; but the mechanism by which this takes place is yet to be understood.

Previous analyses in a variety of organisms (mouse embryonic stem cells (2), yeast (4) and human cell lines (5)) have shown that the composition of ribosomes is heterogeneous. These different ribosome populations may be able to regulate translation. In fact, specialisation of ribosomes is thought to be able to occur through heterogenous ribosomes that contain (a) additional protein components (6), (b) substitution of ribosomal protein (RP) paralogs (7), (c) post-translational modification of RPs (8) and (d) rRNA modifications (9). All these changes to the composition of ribosomes could potentially contribute to functionally specialised ribosomes (10).

Two significant factors have contributed to the logic behind the idea of specialised ribosomes: (a) prevalence of tissue specific RP expression and (b) distinctive phenotypes when RP genes are disrupted (11). Many RPs exhibit differences in expression levels across various tissues in mammals $(1,12,13)$, plants (14) and insects (7). For example, $\mathrm{RpS} 5 \mathrm{~A}$ and $\mathrm{RpS5B}$ are expressed in different cell types during early Arabidopsis thaliana development (15). Disrupted $\mathrm{RP}$ genes result in varied, distinctive phenotypes suggesting that not all ribosomal components are equally important all the time. For example, Rpl38 mouse mutants exhibit a homeotic transformation phenotype with few other effects (1), whilst Rpl38 mutants in D. melanogaster exhibit large wings, small bristles, delayed development and disorganised wing hair polarity (16).

Several human diseases, called ribosomopathies, have been attributed to mutations in RP genes. These diseases exhibit varying clinical symptoms between different RP mutations $(17,18)$. This suggests human RPs may pos-

\footnotetext{
${ }^{*}$ To whom correspondence should be addressed. Tel: +44 113343 9607; Email: j.aspden@leeds.ac.uk

Correspondence may also be addressed to Juan Fontana. Email: j.fontana@leeds.ac.uk

${ }^{\dagger}$ The authors wish it to be known that, in their opinion, the first two authors should be regarded as Joint First Authors.

(C) The Author(s) 2021. Published by Oxford University Press on behalf of Nucleic Acids Research.

This is an Open Access article distributed under the terms of the Creative Commons Attribution License (http://creativecommons.org/licenses/by/4.0/), which permits unrestricted reuse, distribution, and reproduction in any medium, provided the original work is properly cited.
} 
sess specialised functions, through their requirement for the translation of specific mRNA pools, but this could also be the result of extra-ribosomal RP functions or ribosome insufficiency. Mutations in RPS19, RPS28, RPS10 and RPS5 result in the ribosomopathy Diamond-Blackfan anaemia. Knocking down these RPs specifically results in reduced translation of the erythropoietic transcription factor GATA1, whilst the translation of other mRNAs is unaffected (18). Therefore, to properly understand ribosomopathies it is necessary to dissect how differences in RP levels impacts ribosome composition and structure.

Human cytoplasmic ribosomes usually comprise of 80 RPs and 4 rRNAs. This is similar across the majority of multicellular eukaryotes, including $D$. melanogaster with 80 RPs and 5 rRNAs. However, there are 93 cytoplasmic RP genes annotated in FlyBase: 39 small subunit proteins and 54 large subunit proteins (19). These additional genes code for 13 paralogs in D. melanogaster. In fact, many RP genes possess paralogs across eukaryotes, for example human RPL3 and RPL3L (13) and A. thaliana RPS $8 A$ and $R P S 8 B(15)$. In total, there are 19 pairs of paralogs in humans (13) and all 80 RPs in A. thaliana have paralogs (20). Expression analyses at RNA and protein levels have indicated there are differences in levels of both canonical RPs and RP paralogs across tissues $(21,22)$. But given the possibility for extra-ribosomal effects of these differences, the impact on ribosomes cannot be determined without detailed characterisation of ribosomal composition and structure in concert.

To dissect the function of ribosome heterogeneity it is necessary to understand the biological importance within the context of whole organisms and their development. Within the developmental biology field, a large proportion of research on gene expression control focuses on the contribution of transcription. However, during development a variety of processes and key time points have been shown to be highly dependent on the regulation of mRNA translation, e.g. oogenesis in Xenopus (23), early embryo development in Drosophila (24) and mammalian erythropoiesis (25). The balance between self-renewal and differentiation at the stem cell niche is highly dependent on translation in both the ovary and the testis (26). This is exemplified by disruptions to the stem cell niche in the testis when RPs are knocked down, e.g. RpL19 RNAi results in the over-proliferation of early germ cells in D. melanogaster (27). During the meiotic phase of gametogenesis, transcription does not occur, therefore meiotic cells rely on post-transcriptional gene regulation (28). The translational machinery has evolved to become specialised within the testis with various testis-specific components, e.g. eIF4E-3 in D. melanogaster (29). Many of the RP mutants associated with the Minute phenotypes have impaired fertility in both males and females $(30,31)$. To date, a robust assessment of ribosome composition across tissues, including gonads, is missing.

Here, we hypothesise that specialised ribosomes exist in the $D$. melanogaster testis, to provide an additional level of mRNA translational regulation during spermatogenesis. Thus, we set out to determine potential changes to the $D$. melanogaster ribosome by probing the protein composition in three adult tissues (head, testis and ovary) and in embryos. Using quantitative mass spectrometry, we identified heterogeneous ribosome populations, especially in the gonads. The main sources of this variation in ribosome composition are paralog-enrichment and paralog-switching, as evidenced by western blotting, cryo-electron microscopy (cryo-EM) and RNA-Seq analysis. We found little difference in composition between single $80 \mathrm{~S}$ ribosomes and the more translationally active polysome ribosomes from the same tissue. We solved structures of different ribosome populations to understand the potential mechanistic impact of these paralog-switching events, and found structural differences between testis and ovary ribosomes. To understand the broader importance of specialisation through paralogswitching events we analysed the levels of conservation between paralog pairs. We found that RpL22 has a duplicate RPL22L in mammals (including humans), and RpL22-like in Drosophila. These duplication events have occurred independently suggesting that it may represent a common mechanism of specialisation across a range of organisms and ribosomes.

\section{MATERIALS AND METHODS}

\section{Growth conditions}

D. melanogaster wild type (Dahomey) were raised on standard sugar-yeast agar (32). Flies were kept at $25^{\circ} \mathrm{C}$ and $50 \%$ humidity with a 12:12 h light:dark cycle in 6 oz Square Bottom Bottles (Flystuff).

\section{Tissue harvest}

$\sim 300$ pairs of ovaries per replicate were harvested from 3- to 6-day-old females in $1 \times$ PBS (Lonza) with $1 \mathrm{mM}$ DTT (Sigma) and $1 \mathrm{U} / \mu 1$ RNAsin Plus (Promega) and flash frozen in liquid nitrogen. $\sim 500$ (replicate 1 ) and $\sim 1000$ (replicates 2 and 3) pairs of testes were harvested from 1to 4-day-old males in $1 \times$ PBS with $2 \mathrm{mM}$ DTT and $1 \mathrm{U} / \mu \mathrm{l}$ RNAsin Plus and flash frozen in groups of $\sim 10$ pairs. $\sim 500$ heads ( $\sim 50: 50$ female:male, $0-4$ day old) per replicate were isolated by flash freezing whole flies and subjected to mechanical shock to detach heads. Heads were passed through $1 \mathrm{~mm}$ mesh filter with liquid nitrogen and transferred to a Dounce homogeniser for lysis. $\sim 500 \mu 1$ of $0-2 \mathrm{~h}$ embryos per replicate were obtained from cages after pre-clearing for $2 \mathrm{~h}$. Laying plates comprised of 3.3\% agar, $37.5 \%$ medium red grape juice compound (Young's Brew) and 0.3\% methyl 4-hydroxybenzoate, supplemented with yeast paste of active dried yeast (DCL) and dH2O. Embryos were washed in $\mathrm{dH} 20$ and embryo wash buffer $[102.5 \mathrm{mM} \mathrm{NaCl}$ (Sigma), 0.04\% TritonX-100 (Sigma)] and then flash frozen with minimal liquid.

\section{Ribosome purification}

All stages were performed on ice or at $4^{\circ} \mathrm{C}$ wherever possible. Ovaries and testes were disrupted using RNase-free $1.5 \mathrm{ml}$ pestles (SLS) in lysis buffer A [50 mM Tris- $\mathrm{HCl}$ pH 8 (Sigma), $150 \mathrm{mM} \mathrm{NaCl}, 10 \mathrm{mM} \mathrm{MgCl} 2$ (Fluka), 1\% IGEPAL CA-630 (Sigma), $1 \mathrm{mM}$ DTT, $100 \mu \mathrm{g} / \mathrm{ml}$ cycloheximide, $2 \mathrm{U} / \mu 1$ Turbo DNase (Thermo Fisher), $0.2 \mathrm{U} / \mu 1$ RNasin Plus, $1 \times$ EDTA-free protease inhibitor cocktail (Roche)]. Lysis buffer A does not disrupt embryos present 
within the ovary sample, since bleach would be required to remove the chorion. Ovaries and testes were lysed in $500 \mu \mathrm{l}$ lysis buffer A. Heads were lysed using $8 \mathrm{ml}$ Dounce homogeniser with loose pestle in $1.5 \mathrm{ml}$ lysis buffer B $[10 \mathrm{mM}$ Tris- $\mathrm{HCl}$ pH 7.5 (Gibco), $150 \mathrm{mM} \mathrm{NaCl,} 10 \mathrm{mM} \mathrm{MgCl}$, 1\% IGEPAL CA-630, 1\% Triton X-100, 0.5\% sodium deoxycholate (Sigma), 2 mM DTT, $200 \mu \mathrm{g} / \mathrm{ml}$ cycloheximide, $2 \mathrm{U} / \mu \mathrm{l}$ Turbo DNase, $40 \mathrm{U} / \mathrm{ml}$ RNAsin Plus, $1 \times$ EDTAfree protease inhibitor cocktail]. Then $500 \mu$ l aliquots were transferred to $2 \mathrm{ml}$ Dounce with tight pestle and further lysed for approximately 30 strokes. Embryos were ground in liquid nitrogen using pestle and mortar, and added to lysis buffer B. All lysates were lysed for $\geq 30$ minutes with occasional agitation, then centrifuged for $5 \mathrm{~min}$ at 17000 $\times \mathrm{g}$ to remove cell debris. Head and embryo cytoplasmic supernatants were obtained by avoiding both floating fat and insoluble pellet, and repeatedly centrifuged until free of debris.

Cytoplasmic lysates were loaded onto $18-60 \%$ sucrose gradients $(50 \mathrm{mM}$ Tris- $\mathrm{HCl}$ pH 8.0, $150 \mathrm{mM} \mathrm{NaCl}, 10 \mathrm{mM}$ $\mathrm{MgCl}_{2}, 100 \mu \mathrm{g} / \mathrm{ml}$ cycloheximide, $1 \mathrm{mM}$ DTT, $1 \times$ EDTAfree protease inhibitor cocktail) and ultra-centrifuged in a SW40Ti rotor (Beckman) for $3.5 \mathrm{~h}$ at $170920 \times \mathrm{g}$ at $4^{\circ} \mathrm{C}$. Ovary and embryo samples were split across two gradients. Fractions were collected using a Gradient Station (Biocomp) equipped with a fraction collector (Gilson) and Econo UV monitor (BioRad). Fractions containing 80S were combined, as were fractions with polysomes (Supplementary Figure S1A-D). Fractions were concentrated using a $30 \mathrm{kDa}$ column (Amicon Ultra-4 or Ultra-15) at $4{ }^{\circ} \mathrm{C}$ and buffer exchanged $(50 \mathrm{mM}$ Tris- $\mathrm{HCl} \mathrm{pH} 8,150 \mathrm{mM}$ $\mathrm{NaCl}, 10 \mathrm{mM} \mathrm{MgCl}_{2}$ ) until final sucrose $\geq 0.1 \%$. Samples were quantified using Qubit Protein Assay Kit (Invitrogen).

For EDTA treatment experiments polysomes were disrupted by the addition of $30 \mathrm{mM}$ EDTA to the lysis buffer and sucrose gradient. $\mathrm{MgCl}_{2}$ was also omitted from the lysis buffer and sucrose gradient.

\section{TMT labelling and high $\mathrm{pH}$ reversed-phase chromatography}

An equal amount $(\mathrm{TMT} 1=40 \mu \mathrm{g}, \mathrm{TMT} 2=40 \mu \mathrm{g}$, TMT3 $=35 \mu \mathrm{g})$ of each sample was digested with trypsin $(2.5 \mu \mathrm{g}$ trypsin per $100 \mu \mathrm{g}$ protein; $37^{\circ} \mathrm{C}$, overnight), labelled with Tandem Mass Tag (TMT) 6 or 10 plex reagents according to the manufacturer's protocol (Thermo Fisher Scientific) and the labelled samples pooled.

$100 \mu \mathrm{g}$ aliquots of pooled samples were evaporated to dryness, resuspended in 5\% formic acid and then desalted using a SepPak cartridge according to the manufacturer's instructions (Waters). Eluates from the SepPak cartridge was again evaporated to dryness and resuspended in buffer $\mathrm{C}(20 \mathrm{mM}$ ammonium hydroxide, $\mathrm{pH} 10)$ prior to fractionation by high $\mathrm{pH}$ reversed-phase chromatography using an Ultimate 3000 liquid chromatography system (Thermo Scientific). In brief, samples were loaded onto an XBridge BEH C18 Column (130 A, $3.5 \mu \mathrm{m}, 2.1 \mathrm{~mm} \times 150 \mathrm{~mm}$, Waters) in buffer $\mathrm{C}$ and peptides eluted with increasing gradient of buffer D $(20 \mathrm{mM}$ ammonium hydroxide in acetonitrile, $\mathrm{pH} 10$ ) from 0 to $95 \%$ over $60 \mathrm{~min}$. The resulting fractions were evaporated to dryness and resuspended in $1 \%$ formic acid prior to analysis by nano-LC-MS/MS using an
Orbitrap Fusion Tribrid mass spectrometer (Thermo Scientific).

\section{Nano-LC mass spectrometry}

High pH RP fractions were further fractionated using an Ultimate 3000 nano-LC system in line with an Orbitrap Fusion Tribrid mass spectrometer (Thermo Scientific). In brief, peptides in 1\% (vol/vol) formic acid were injected onto an Acclaim PepMap C18 nano-trap column (Thermo Scientific). After washing with $0.5 \%$ (vol/vol) acetonitrile $0.1 \%$ (vol/vol) formic acid peptides were resolved on a 250 $\mathrm{mm} \times 75 \mu \mathrm{m}$ Acclaim PepMap C18 reverse phase analytical column (Thermo Scientific) over a 150 minute organic gradient, using seven gradient segments $(1-6 \%$ solvent $\mathrm{B}$ over $1 \mathrm{~min}, 6-15 \%$ B over $58 \mathrm{~min}, 15-32 \%$ solvent B over $58 \mathrm{~min}$, $32-40 \%$ solvent B over $5 \mathrm{~min}, 40-90 \%$ solvent B over $1 \mathrm{~min}$, held at $90 \%$ solvent $\mathrm{B}$ for $6 \mathrm{~min}$ and then reduced to $1 \%$ solvent B over $1 \mathrm{~min}$ ) with a flow rate of $300 \mathrm{nl} \mathrm{min}^{-1}$. Solvent $\mathrm{B}$ was aqueous $80 \%$ acetonitrile in $0.1 \%$ formic acid. Peptides were ionized by nano-electrospray ionization at $2.0 \mathrm{kV}$ using a stainless-steel emitter with an internal diameter of $30 \mu \mathrm{m}$ (Thermo Scientific) and a capillary temperature of $275^{\circ} \mathrm{C}$.

All spectra were acquired using an Orbitrap Fusion Tribrid mass spectrometer controlled by Xcalibur 2.1 software (Thermo Scientific) and operated in data-dependent acquisition mode using an SPS-MS3 workflow. FTMS1 spectra were collected at a resolution of 120000 with an automatic gain control (AGC) target of 200000 and a max injection time of $50 \mathrm{~ms}$. Precursors were filtered with an intensity threshold of 5000, according to charge state (to include charge states 2-7) and with monoisotopic peak determination set to peptide. Previously interrogated precursors were excluded using a dynamic window (60 s $\pm 10 \mathrm{ppm})$. The MS2 precursors were isolated with a quadrupole isolation window of $1.2 \mathrm{~m} / z$. ITMS2 spectra were collected with an AGC target of 10000 , max injection time of $70 \mathrm{~ms}$ and CID collision energy of $35 \%$.

For FTMS3 analysis, the Orbitrap was operated at 50000 resolution with an AGC target of 50000 and a max injection time of $105 \mathrm{~ms}$. Precursors were fragmented by high energy collision dissociation (HCD) at a normalised collision energy of $60 \%$ to ensure maximal TMT reporter ion yield. Synchronous Precursor Selection (SPS) was enabled to include up to $5 \mathrm{MS} 2$ fragment ions in the FTMS3 scan.

\section{TMT data analysis}

The raw data files were processed and quantified using Proteome Discoverer software v2.1 (Thermo Scientific) and searched against the UniProt $D$. melanogaster database (downloaded March 2018; 41,157 sequences) using the SEQUEST HT algorithm. Peptide precursor mass tolerance was set at $10 \mathrm{ppm}$, and $\mathrm{MS} / \mathrm{MS}$ tolerance was set at 0.6 Da. Search criteria included oxidation of methionine $(+15.995 \mathrm{Da})$ and acetylation of the protein $\mathrm{N}$ terminus $(+42.011 \mathrm{Da})$ as variable modifications and carbamidomethylation of cysteine $(+57.021 \mathrm{Da})$ and the addition of the TMT mass tag $(+229.163 \mathrm{Da})$ to peptide N-termini and lysine as fixed modifications. Searches were 
performed with full tryptic digestion and a maximum of two missed cleavages were allowed. The reverse database search option was enabled and all data was filtered to satisfy false discovery rate (FDR) of 5\% (33) and reported.

Peptide IDs not corresponding to D. melanogaster proteins were removed from all TMT replicates. Using the protein grouping decided by PD2.1, master protein selection was improved using an in-house script to select the UniProt accession (database downloaded January 2021; 42818 sequences) with the best annotation whilst maintaining confidence in protein identification and quantitation. This resulted in a list of 1906 proteins for TMT1, 3613 proteins for TMT2 and 1869 proteins for TMT3. Abundances are the sum of the $\mathrm{S} / \mathrm{N}$ values for the TMT reporter groups for all peptide-spectrum matches (PSMs) matched to the protein. Normalised abundances of these values were obtained by normalising the Total Peptide Amount in each sample such that the total signal from each TMT tag is the same. Scaled abundances are either normalised abundances scaled to a pooled sample or the normalised abundance scaled to the average of all samples within that replicate. Data from all three replicates was merged into one data sheet from which comparisons between tissues were made with statistical analyses for all proteins detected (Supplementary Table S1).

Pair-wise comparisons were made to calculate the $\log _{2}$ fold-change difference of proteins across different tissue samples. For TMT1 and 3, normalised abundances were used, and for TMT2 the normalized abundance was scaled to a pooled sample to allow comparison between TMT runs. Standard t-test was used to test the statistical significance of the $\log _{2}$ fold-changes between tissue samples. Analysis of TMT data and hierarchical clustering were performed in R.

Fold-change differences between highly similar peptides were determined to estimate relative abundances of different RP paralog pairs between tissue samples. PSMs were only used for this purpose if the peptides were of identical length and had fewer than two amino acid changes.

\section{Antibodies and western blotting}

RP paralog specific antibodies were generated using custom peptides for RpL22 (CNKGDTKTAAAKPAEK), RpL22-like (CSSQTQKKNASKAKSK), RpS19a (CQ IVFKQRDAAKQTGP), RpS19b (CKQRERSAPVSMII TT), RpL37a (CREGTQAKPKKAVASK) and RpL37b (CRNGLREGGAAKKKTN) (Pepceuticals, UK). RpL22 and RpL22-like antibodies were isolated from serum via affinity purification using HiTrap NHS-activated HP columns (Cytiva Life Sciences). RpS5a and RpS5b antibodies were kindly gifted by the Lasko lab (34).

Protein samples were separated on a 4-20\% Mini-Protean TGX gel before being transferred to $0.2 \mu \mathrm{m}$ nitrocellulose membrane, which was blocked for $1 \mathrm{~h}$ in $5 \%$ milk in $1 \times$ TBST. Membranes were probed with antibodies diluted in $1 \times$ TBST. Primary antibody concentrations were: RpL22 1:2500, RpL22-like 1:2500, RpS5a 1:1000, RpS5b 1:1000, RpS19a 1:25, RpS19b 1:25, RpL37a 1:25, RpL37b 1:25, RpL40 1:1000 (ab109227, abcam).

\section{Source of RNA-Seq data}

RNA-Seq data was extracted from ModMine (intermine.modencode.org) (35) with data from modENCODE project $(36,37)$. Values are Reads Per Kilobase of transcript, per Million mapped reads (RPKMs).

\section{Cryo-EM}

400 mesh copper grids with a supporting carbon lacey film coated with an ultra-thin carbon support film $<3 \mathrm{~nm}$ thick (Agar Scientific, UK) were employed. Grids were glowdischarged for $30 \mathrm{~s}$ (easiGlow, Ted Pella) prior to applying 3 $\mu l$ of purified ribosomes, and vitrification was performed by plunge-freezing in liquid ethane cooled by liquid nitrogen using a Leica EM GP device (Leica Microsystems). Samples were diluted using the buffer exchange buffer $(50 \mathrm{mM}$ Tris- $\mathrm{HCl} \mathrm{pH} \mathrm{8,150} \mathrm{mM} \mathrm{NaCl}, 10 \mathrm{mM} \mathrm{MgCl}$ ) as required. Cryo-EM data was collected on a FEI Titan Krios (Astbury Biostructure Laboratory, University of Leeds) EM at $300 \mathrm{kV}$, using a total electron dose of $80 \mathrm{e}^{-} / \AA^{2}$ and a magnification of $75000 \times$ at -2 to $-4 \mu \mathrm{m}$ defocus. Movies were recorded using the EPU automated acquisition software on a FEI Falcon III direct electron detector, in linear mode, with a final pixel size of $1.065 \AA$ /pixel (Supplementary Table S2).

\section{Image processing}

Initial pre-processing and on-the-fly analysis of data was performed as previously described (38). Image processing was carried out using RELION 2.0/2.1 or 3.0 (39). MOTIONCOR2 (40) was used to correct for beam-induced motion and calculate averages of each movie. gCTF (41) was used for contrast transfer function determination. Particles were automatically picked using the Laplacian of Gaussian function from RELION (42). Particles were classified using two rounds of reference-free 2D classification. For the testis $80 \mathrm{~S}$ reconstruction, particles contributing to the best 2D class averages were then used to generate an initial 3D model. For the ovary $80 \mathrm{~S}$ and testis polysome reconstruction, the testis $80 \mathrm{~S}$ average was used as initial reference. Particles were classified by two rounds of $3 \mathrm{D}$ classification, and the best 3D classes/class were 3D refined, followed by perparticle CTF correction and Bayesian polishing (42). Postprocessing was employed to mask the model, and to estimate and correct for the $B$-factor of the maps (43). The testis $80 \mathrm{~S}$ map was further processed by multi-body refinement, as previously described (44). The final resolutions were determined using the 'gold standard' Fourier shell correlation $(\mathrm{FSC}=0.143)$ criterion (Supplementary Table S2). Local resolution was estimated using the local resolution feature in RELION.

\section{Atomic modelling}

The D. melanogaster embryo ribosome (PDB code: 4V6W) was used as a model to calculate the structures of the testis and ovary ribosomes. First, the full atomic model was fitted into the testis $80 \mathrm{~S}$ cryo-EM average using the 'fit in map' tool from Chimera (45). Then, fitting was refined by rigidbody fitting individual protein and RNA PDBs into the 
maps using Chimera. The $18 \mathrm{~S}$ and $28 \mathrm{~S}$ ribosomal RNAs were split into two separate rigid bodies each. Proteins and RNAs not present in our averages (i.e. elongation factor 2 and Vig2 for all models, and E-tRNA) and proteins and RNA with poor densities (i.e. RpLP0 and RpL12, and some regions of the $18 \mathrm{~S}$ and $28 \mathrm{~S}$ ribosomal RNAs) were removed at this stage. The paralog proteins used for each ribosome are listed in Table 1. For the testis $80 \mathrm{~S}$ atomic model, IFRD1 was modelled using SWISS-MODEL (46), based on the atomic model for rabbit IFRD2 (PDB model: 6MTC). For the testis polysome model, the mRNA was based on PDB model $6 \mathrm{HCJ}$, and the P-tRNA on the E-tRNA from PDB model 4V6W. The full atomic models were refined using Phenix (47), and the paralogs listed in Figure 2A (plus RpL31 and RpS18) were manually inspected and corrected using COOT (48) (except Rp10Ab, which was not manually inspected due to the low resolution of that area in the average maps, and RpLP0, which was not present in the model). This cycle was repeated at least three times per ribosome model. The quality of the atomic models was assessed using the validation server from the PDB website (https://validate-pdbe.wwpdb.org/). As the 60S acidic ribosomal protein P0 deposited (RpLP0) in the PDB (4V6W) is from Homo sapiens, we generated a D. melanogaster homology model using SWISS-MODEL. This protein was rigidbody fitted using Chimera after the atomic model refinement and is displayed in Figure 4 for relative position and size comparison purposes only. Figures were generated using Chimera.

\section{Vertebrate dataset construction}

Coding DNA sequence (CDS) data for 207 vertebrate animals and four non-vertebrates (D. melanogaster, two Caenorhabditis species and $S$. cerevisiae) were obtained from Ensembl (release 97, (49)). We performed homology searches using two human RpL22 family proteins (RPL22 and RPL22L) against 6922005 protein sequences using BLASTp $\left(\mathrm{e}^{-5}\right)$ (50). We identified 1,082 potential RpL22 proteins from 185 vertebrates and four non-vertebrates, which were homologous to one or both human RpL22 proteins. As an initial step to reduce the amount of redundancy in the vertebrate dataset, 181 potential RpL22 proteins from 42 selected vertebrates (including humans) were retained to represent as broad a taxonomic sampling of the group. All non-vertebrate sequences, with the exception of two $S$. cerevisiae RpL22 proteins (RPL22A and RPL22B), were also removed from the dataset. Ninety-two alternative transcripts and spurious hits were removed from the dataset through manual cross-validation with Ensembl Genome Browser to give a total of 87 vertebrate and 2 yeast RpL22 family proteins.

\section{Invertebrate dataset construction}

A CDS dataset for 78 invertebrate animals was obtained from Ensembl Metazoa (release 44, (49)). The sequence homology search was performed using two $D$. melanogaster RpL22 family proteins (RPL22 and RPL22like) were searched against 1618385 protein sequences using BLASTp $\left(\mathrm{e}^{-5}\right)(50)$. BLASTp identified 90 potential
RpL22 family proteins across 70 invertebrates, which were homologous to one or both $D$. melanogaster RpL22 proteins. Fifteen alternative transcripts and spurious hits were removed from the dataset through manual cross-validation with Ensembl Genome Browser to give a total of 75 invertebrate RpL22 family proteins. Together with 87 vertebrate and 2 outgroup proteins, our final dataset consisted of 164 $\mathrm{RpL} 22$ family proteins sampled across the metazoan tree of life.

\section{Phylogenetic reconstructions of metazoan RpL22 family}

Initial phylogenetic reconstruction of the metazoan RpL22 family was performed using the full dataset of 164 sequences (87 invertebrate sequences, 75 vertebrate sequences and two yeast sequences). All sequences were aligned using three different alignment algorithms: MUSCLE (51), MAFFT (52) and PRANK (53). MUSCLE was run with the default parameters, and MAFFT was run with the automaticallyselected most-appropriate alignment strategy (in this case, L-INS-I). PRANK was run with both the default parameters and the PRANK+F method with 'permanent' insertions. All four resultant alignments were compared against each other using MetAl (54), and were all judged to be mutually discordant based on differences of $20-25 \%$ between each pair of alignments. Column-based similarity scores were calculated for each alignment using the norMD statistic (55). The MUSCLE alignment had the highest columnbased similarity score (1.281) and was selected for further analysis. This alignment was trimmed using TrimAl's gappyout method (56). Maximum-likelihood phylogenetic reconstruction was performed on the trimmed alignment using IQTREE (57), with a WAG+R6 model selected by ModelFinder Plus (58) and 100 bootstrap replicates.

A smaller, taxonomically-representative $R p L 22$ family dataset containing 50 RpL22 genes from 30 animals and $S$. cerevisiae was constructed for a representative RpL22 family phylogeny. This dataset was aligned using the same four methods described above, and all alignments were judged to be mutually discordant (differences of 19-37\%) using MetAl (54). The MUSCLE alignment had the highest column-based similarity score assigned by norMD (0.702) and was selected for further analysis. As above, this alignment was trimmed using TrimAl's gappyout method. Maximum-likelihood phylogenetic reconstruction was performed on the trimmed alignment using IQTREE (57), with a DCMut+R3 model selected by ModelFinder Plus (58) and 100 bootstrap replicates.

\section{RESULTS}

Heterogeneous ribosome populations exist in different tissues

Many eukaryotic genomes contain numerous RP paralogs yet their contribution to ribosomal function is poorly understood. In D. melanogaster there are 93 RP genes (FlyBase), which include 13 pairs of paralogs, resulting in 80 proteins in each ribosome (59). The expression of RPs and specifically RP paralogs has been reported to vary in a tissue specific manner $(15,21)$. To profile potential differences in expression in $D$. melanogaster we analysed publicly available RNA-Seq data across various developmen- 
tal time points and tissues. Hierarchical clustering of RP mRNA abundances across these different biological samples reveals variations in expression of RP mRNAs between tissues, with a cluster of RPs with much higher expression in the testis compared to other tissues (Figure 1A). This includes $R$ pl22-like, a paralog of $R p l 22$ previously reported as a testis-specific ribosomal protein (7). This result suggests the presence of testis-specific translational machinery.

To determine whether these different RPs are translated and incorporated into ribosomes we assessed the protein composition of ribosomes from testes, ovaries, heads (mixture of male and female) and embryos ( $0-2 \mathrm{~h})$. Ribosomal complexes were purified using sucrose gradients and ultracentrifugation (Figure 1B). Both 80S (monosome) and polysome complexes were isolated. The relative amounts of ribosomes existing as $80 \mathrm{~S}$ or polysome complexes varied substantially across the samples (Supplementary S1). In general, polysome levels are substantially lower than monosome levels in tissues (Supplementary S1), as previously shown in vivo (60), especially when compared to cultured cells (61). Both monosome and polysome fractions were isolated for each tissue and subjected to quantitative mass spectrometry (tandem mass tag; TMT). Overall correlation between the biological replicates is high as RPs in testis $80 \mathrm{~S}$ samples had Pearson's correlation coefficients of 0.96, 0.95 and 0.96 (Figure 1C, Supplementary S2). Similar results are obtained when considering only ribosomal paralogs and across samples (Supplementary S2). We only used unique peptides in our analysis, which is particularly important when distinguishing between paralogs (Supplementary S3 and S4).

To understand differences in ribosome composition in $80 \mathrm{~S}$ complexes between the tissues, protein abundances of RPs were subject to hierarchical clustering (Figure 1D). Two clear protein clusters emerged. One where proteins are enriched in the testis $80 \mathrm{~S}$ ribosomes compared to $80 \mathrm{~S}$ ribosomes from other tissues, including RpL22-like, RpL37b, RpS19b, RpS10a and RpS28a, RpS15Ab. The other is an ovary $80 \mathrm{~S}$ enriched cluster of ribosomal proteins, including RpS5b, RpL24-like, RpL7-like and RpL0-like (Figure 1D). Similar clusters are seen for the three biological repeats (Figure 1D, Supplementary S5A and B).

To identify substantial and statistically significant differences in ribosomal protein composition, differences in abundances were plotted between different $80 \mathrm{~S}$ complexes, employing 1.5 -fold-change and $0.05 P$-value as cut-offs. Comparison of testis $80 \mathrm{~S}$ with head $80 \mathrm{~S}$ and ovary $80 \mathrm{~S}$ revealed that the same six RPs (RpL22-like, RpL37b, RpS19b, RpS10a, RpS28a and RpS15Ab) are highly enriched in the testis $80 \mathrm{~S}$ (Figure $1 \mathrm{E}$ and $\mathrm{F}$ ). Likewise, comparison of ovary $80 \mathrm{~S}$ with testis $80 \mathrm{~S}$ and head $80 \mathrm{~S}$ revealed that the same 4 RPs (RpS5b, RpL24-like, RpL7-like and RpL0-like) are enriched in ovary $80 \mathrm{~S}$ (Figure $1 \mathrm{~F}$ and $\mathrm{G}$ ). Additionally, the comparison between ovary $80 \mathrm{~S}$ with head $80 \mathrm{~S}$ revealed that RpS12 and RACK1 are also enriched in ovary $80 \mathrm{~S}$ compared to head $80 \mathrm{~S}$. The comparisons involving head $80 \mathrm{~S}$ showed an enrichment of RpS11 in the head 80S (Figure 1E and G). Embryo 80S showed no paralog enrichment (Supplementary S5C-E). Overall, heterogeneity seems most common in the gonads and we identify both testis- and ovary-enriched RPS.

\section{Ribosomal protein paralogs contribute to ribosome hetero- geneity}

There are 13 pairs of RP paralogs in the D. melanogaster genome and from our TMT data we can see the majority are both expressed and incorporated into $80 \mathrm{~S}$ ribosomes in at least one of the analysed tissues. In fact, out of the enriched RPs we identified, 10 out of 13 were paralogs (Figure $1 \mathrm{E}-\mathrm{G})$. Hierarchical clustering of just RP paralogs reemphasises the existence of gonad specific ribosomal complexes mostly through changes to RP paralogs (Figure 2A). To understand the relationship between each of the two paralogs we compared the quantitative differences in abundances between tissues for each paralog pair (i.e. $\log _{2}$ foldchange $-\log _{2} \mathrm{FC}-$ between ovary and testis, Figure $2 \mathrm{~B}$ ). $\mathrm{RpS14a}$ and $\mathrm{RpS14b}$ have the same amino acid sequences, so are indistinguishable by mass spectrometry and therefore they were excluded from this analysis. In addition, RpL10Aa was also omitted because no unique peptides were detected. Calculating $\log _{2} \mathrm{FC}$ between ovary and testis $80 \mathrm{~S}$ complexes indicates that out of 11 pairs we could analyse in this way, the six testis-specific paralogs are enriched in the testis and the four ovary-specific paralogs are enriched in the ovary. Only for the RpL34 paralog pair did neither paralog show differential incorporation into the $80 \mathrm{~S}$ ribosome between ovary and testis. Overall, only one paralog from each pair is significantly enriched in either ovary or testis, whilst the other paralog in each pair shows little or no difference (i.e. $\log _{2} \mathrm{FC}$ below 0.5 ). In general, these other paralogs are slightly enriched in the other tissue but not significantly. For example, for the paralog pair RpS5, RpS5b is enriched in the ovary $(P$ value $=0.02)$ and $\mathrm{RpS5a}$ is more enriched in the testis $(P$ value $=0.026)$.

To further compare across all the tissues in this way we calculated $\log _{2} \mathrm{FC}$ of embryo, ovary and testis to head, because head showed little heterogeneity (Figure 2C). This analysis shows the same testis-specific and ovaryspecific paralogs (except for RpS5, which is not significant in this analysis). Interestingly $\mathrm{RpS} 5 \mathrm{~b}$ is enriched in embryo, ovary and testis, when compared to the head, but is not statistically significant. Thus, RpS5b has an unusually broad incorporation across the different sampled ribosomes.

To validate the difference in paralog levels seen by TMT we generated paralog specific antibodies for RpL22, RpL22-like, RpL37a, RpL37b, RpS19a and RpS19b. Using these and published RpS5a and RpS5b antibodies (34) we probed paralog levels in purified $80 \mathrm{~S}$ ribosomes isolated from ovaries, testes and heads. This analysis confirmed that RpL22-like is enriched in the testis when compared to ovary and head $80 \mathrm{~S}$ ribosomes (Figure 2D). Furthermore, it showed a concomitant reduction of RpL22 levels in testis $80 \mathrm{~S}$, the extent of which indicates that a larger proportion of the testis $80 \mathrm{~S}$ ribosomes contain $\mathrm{RpL} 22$-like rather than RpL22. A similar pattern was seen for RpL37b and RpL37a, again suggesting that a larger proportion of the testis $80 \mathrm{~S}$ ribosomes contain RpL37b rather than RpL37a. $\mathrm{RpS19b}$ was also detected in testis $80 \mathrm{~S}$ ribosomes, but without such a large reduction in $\mathrm{RpS19a}$ when compared to ovary and head. This suggests that although RpS19b is enriched in the testis, it is present in a similar proportion of 
A

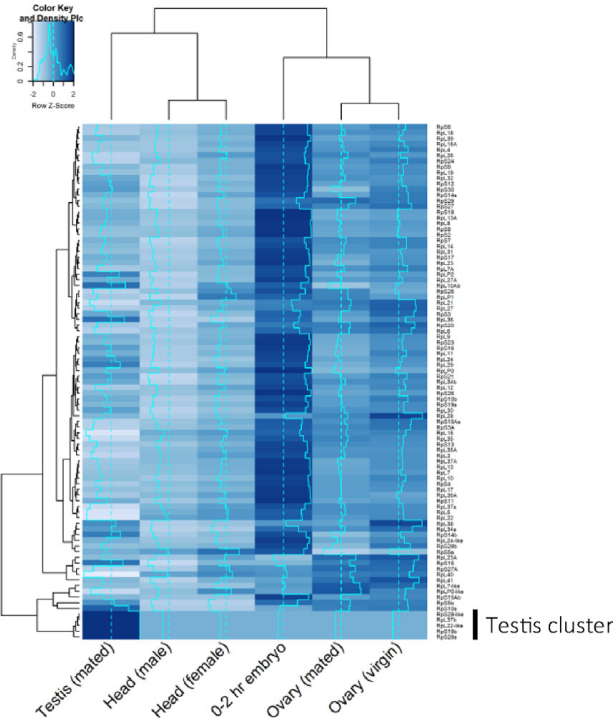

D
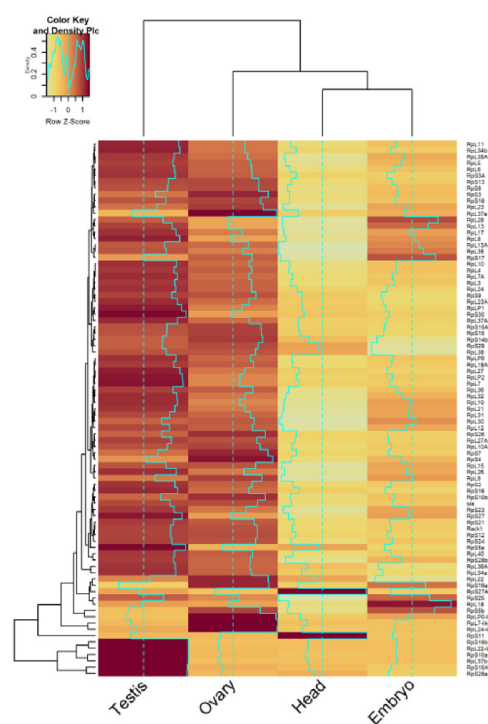

I Ovary cluster Testis cluster

$\mathbf{F}$

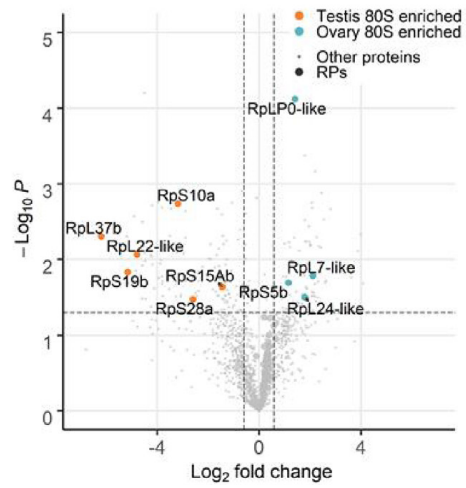

B

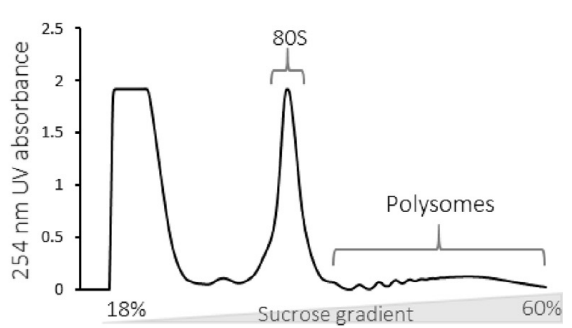

C

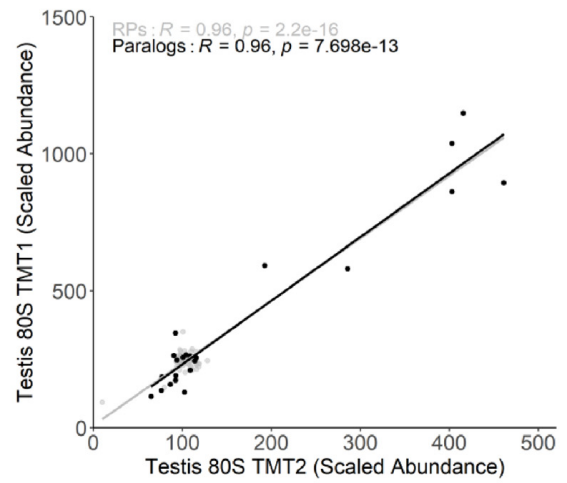

E

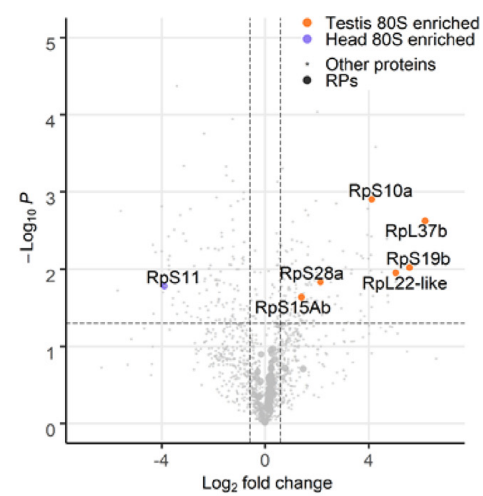

G

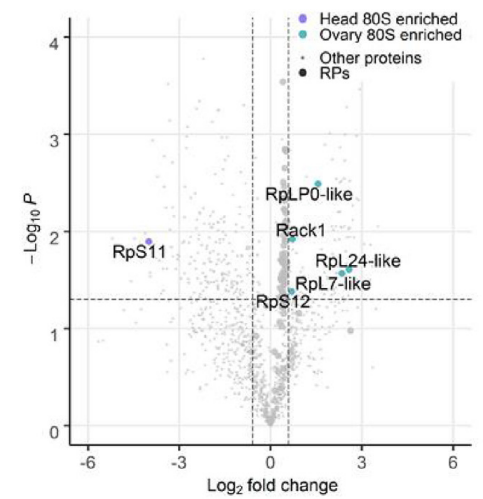

Figure 1. Heterogeneous ribosome populations exist in different tissues. (A) Hierarchical clustering of modENCODE RNA-Seq data (RPKM) for ribosomal proteins (RPs) across testis, ovary, head and embryo reveals differences in RP expression clustered according to row i.e. ribosomal protein with Z-scores calculated and plotted. The analysis shows a cluster of 5 testis-specific RPs. (B) Schematic of strategy used to isolate and compare $80 \mathrm{~S}$ ribosome and polysome complex composition. (C) Correlation of two biological repeats of TMT mass spectrometry experiments for scaled protein abundances within $80 \mathrm{~S}$ ribosomes isolated from testis shows replicates are reproducible. Correlations are shown for all RPs (grey) and RP paralogs (black) with Pearson's correlation coefficients calculated. (D) Hierarchical clustering of $\log _{2}$ scaled protein abundances from replicate 2 . Normalised abundances were scaled to a control pool from which $Z$-scores were calculated and plotted. Heatmap is clustered according to row i.e. ribosomal protein. (E-G) Volcano plots highlighting the differences in ribosomal protein composition between (E) head and testis, (F) testis and ovary, and (G) ovary and head tissues. Enriched proteins have a $>1.5$-fold change with a $P$-value of $<0.05$. Enriched RPs are labelled. 
A

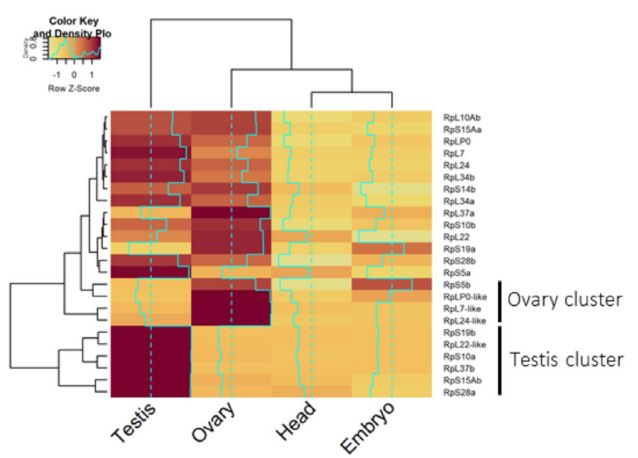

C

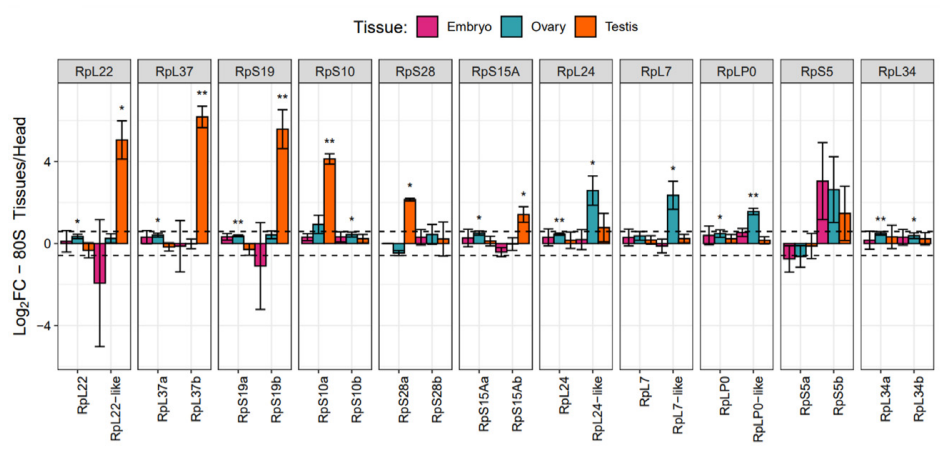

B

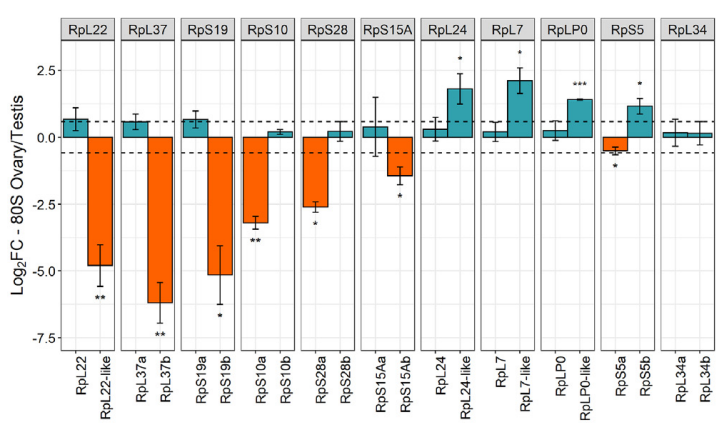

Figure 2. Gonad ribosome heterogeneity through paralog enrichment and paralog-switching. (A) Hierarchical clustering of $\log _{2}$ scaled abundances for the

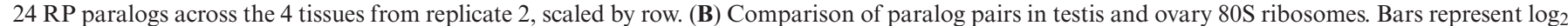
fold-change between the two tissues. Dotted lines represent a $\log _{2}$ fold-change of $1.5,{ }^{*} P$-value $<0.05, * *<0.01, * * *<0.001$. (C) Comparison of paralog pairs in all tissues relative to head tissue. Dotted lines represent a $\log _{2}$ fold-change of $1.5,{ }^{*} P$-value $<0.05, * *<0.01, * * *<0.001$. (D) Immunoblots of purified $80 \mathrm{~S}$ monosomes from ovary, testis and heads tissues using paralog specific antibodies. (E, F) Comparison of highly similar unique peptides for (E) $\mathrm{RpS19a/b}$ and (F) RpS5a/b in testis, ovary, and head tissues. Peptides compared for each paralog pair are of the same size and have 1 or 2 amino acid changes (differences shown in the key within square brackets). Comparisons are shown as $\log _{2}$ fold-change differences for each paralog pair. (G) $\log _{2}$-fold difference plot comparing testis and ovary at both RNA (ovary RNA, mated females) and protein levels. All 85 RPs detected in our TMT experiments are plotted with testis/ovary specific paralogs labelled. Error bars represent standard deviations. Grey dotted line represents expected relationship between fold-change in RNA and protein. 
Nucleic Acids Research, 2021

ribosomes than RpS19a. The westerns also confirmed the enrichment of RpS5b in the ovary and testis compared with head. This is accompanied by a concomitant reduction of RpS5a in both tissues. Additionally, there is a larger proportion of ovary $80 \mathrm{~S}$ ribosomes that contain $\mathrm{RpS} 5 \mathrm{~b}$ instead of $\mathrm{RpS5a}$.

To provide a more accurate comparison of protein levels between each pair of paralogs a detailed analysis of peptides resulting from the paralogs was performed. Pairs of unique peptides in the TMT data were identified, which corresponded to almost identical parts of the two paralogs but were different by 1 or 2 amino acids. These peptides were different enough that they could be confidently assigned to one of the two paralogs e.g.[D]IDQH[A]VTK from RpS19b and [E]IDQH[V]VTK from RpS19a, but similar enough that the quantification can be directly compared. Only similar peptides with comparable characteristics (e.g. charge and length) can be compared by TMT; therefore we reasoned that highly homologous peptides with only one or two amino acid changes qualified for this criteria. Differences in levels were calculated for each pair for each replicate. For some paralog pairs there were several such peptides that could be analysed whereas for other paralogs, only 1 peptide fitted these parameters. Additionally, some paralogs did not contain unique peptides that allowed for this analysis; these included RpL22 and RpL22-like; and $\mathrm{RpL} 37 \mathrm{a}$ and $\mathrm{RpL} 37 \mathrm{~b}$. This analysis revealed that the fold difference between $\mathrm{RpS} 19 \mathrm{~b}$ and $\mathrm{a}$, is $\sim 0$ in testis, indicating that around equal levels of the two paralogs are present within testis $80 \mathrm{~S}$ ribosomes (Figure 2E). Whereas in ovary and head there is $>16$ times more RpS19a than there is RpS19b. This analysis confirmed paralog switching for RpS5b in ovary (Figure $2 \mathrm{~F}$ ), i.e. there is $\sim 2-8$-fold more RpS5b in ovary $80 \mathrm{~S}$ ribosomes than RpS5a. Furthermore, in the testis $80 \mathrm{~S}$ ribosomes the fold difference suggests there are near equal quantities of $\mathrm{RpS} 5 \mathrm{~b}$ and $\mathrm{RpS} 5 \mathrm{a}$ present (Figure 2F). We note these results correlate with the western blots for the RpS19a/b and RpS5a/b paralogs pairs (Figure 2D). Similar analysis for RpS15Ab/a indicates that although $\mathrm{RpS} 15 \mathrm{Ab}$ is enriched in the testis $80 \mathrm{~S}$ ribosomes, it still represents a minor part of the population compared to RpS15Aa (Supplementary S6A). For both RpS10b/a (Supplementary S6B) and RpS28b/a (Supplementary S6C) only 1 peptide pair could be used for this analysis, and these peptides were not detected in all replicates, therefore we are unable to draw such clear conclusions. However, this data does suggest that even though RpS10a and $\mathrm{RpS} 28 \mathrm{a}$ are enriched in testis $80 \mathrm{~S}$ ribosomes they are both present at relatively low levels compared to RpS10b and $\operatorname{RpS} 28 b$.

In summary these data indicate that there are six paralogs are enriched in testes ribosomes and four in ovaries; and that some of these alternative paralogs are present in a larger proportion of ribosomes than the canonical paralog (i.e. RpL22-like, RpL37b in testis $80 \mathrm{~S}$ and RpS5b in ovary $80 \mathrm{~S})$. Furthermore, RpS19b and RpS5b are present at similar levels to their canonical paralogs in the testis 80S. Together this suggests that there is RP paralog switching occurring in the gonads, whereby the canonical paralog is switched for an alternative one in the majority of ribosomes.
Differences in ribosome composition are not simply the result of expression differences

To understand the expression of RP paralogs, we analysed RNA levels of each of the paralog pairs (Supplementary S7) from published RNA-Seq datasets of the tissues we performed TMT in (35-37). RNA-Seq levels for several of the gonad-enriched paralogs are similar to, or above levels of the canonical paralog in each pair. For example, this is the case for Rpl22-like, Rpl37b, Rps19b, Rps10a, Rps5b in the testis and Rp5b in the ovary (Supplementary S7). Therefore, this RNA-Seq data supports the finding that several paralogs can be considered more than simply testis or ovaryenriched, likely present in a substantial proportion of ribosomes and potentially gonad-switched.

Differences in RNA-Seq levels between testis and ovary were then compared to differences in TMT abundance to examine if paralog enrichment is simply the result of differential expression. An increased RNA expression for a given paralog was generally associated with larger $\log _{2}$ FC in paralog abundance with the $80 \mathrm{~S}$ ribosome (e.g. RpS19b and RpS28a for testis; and RpS5b and RpLP0-like for ovaries; Figure 2G). However, it is clear that differences in protein composition of ribosomes are not simply driven by transcriptional control of paralog genes. Specifically, we identified several instances when RP incorporation into the ribosome does not correlate with mRNA expression level (Figure 2G). For example, Rpl24-like is transcribed at similar levels in ovary and testis (Supplementary S7) but RpL24like is far more abundant in ovary $80 \mathrm{~S}$ than testis $80 \mathrm{~S}$ ribosomes (Figure 2G). The opposite is seen for RpS15Ab, whose RNA levels are similarly low between testis and ovary but is preferentially incorporated into testis 80S (Supplementary S7 and Figure 2G). In general, differences in RP paralog enrichment in tissues are driven by differences in mRNA expression, however, additional regulation is taking place for some RPs, such as RpL24-like and RpS15Ab.

\section{Composition of $80 \mathrm{~S}$ ribosomes and polysomal ribosomes is similar}

There is conflicting evidence as to the functionality or translational activity of monosomes ( $80 \mathrm{~S}$ ribosomes). Some studies suggest that these ribosomes are actively translating (62) whilst others suggest that not all $80 \mathrm{~S}$ ribosomes are engaged in active translation (63). To determine if there was any difference in ribosome composition between monosomes and polysome complexes for a given tissue, we compared the two by TMT. In general, there is very little difference in RP composition between $80 \mathrm{~S}$ ribosomes and polysomes (Supplementary S8). No differences were found between embryo $80 \mathrm{~S}$ and embryo polysomes (Supplementary S8A). However, RpL7-like and RpL24-like are enriched in testis 80S (Supplementary S8B) and RpL24-like is enriched in head polysomes when compared to head $80 \mathrm{~S}$ ribosome complexes (Supplementary S8C). Furthermore, a non-paralog ribosomal protein, RpL38, is enriched in ovary polysomes (Supplementary S8D). Overall, we found larger differences in protein composition between different tissues, than between $80 \mathrm{~S}$ and polysomal ribosomes from the same tissues, and no consistent differences between $80 \mathrm{~S}$ and polysome ribosomes. Together this suggests that from 
the perspective of ribosomal protein composition, there is little difference between $80 \mathrm{~S}$ and polysomal ribosome complexes.

\section{Paralog enrichment is within ribosomal complexes}

To ensure that the complexes we have analysed represent ribosomes, rather than other large protein complexes we assessed the sensitivity of ovary ribosomal complexes to EDTA. EDTA chelates $\mathrm{Mg}^{2+}$ and therefore causes ribosomes to disassociate into $40 \mathrm{~S}$ and $60 \mathrm{~S}$ subunits. The distribution of RPs across sucrose gradients in presence and absence of EDTA was assessed by western blots. These reveal a shift of RPs towards 40S and 60S subunit fractions (Supplementary S9), suggesting that their presence in the complexes we purified was the result of ribosomes. Specifically, RpL22, which is highly abundant in ovary ribosomes, showed a shift from large complexes in the sucrose gradient (polysomes) to small complexes (including 60S subunits) in the presence of EDTA. This was also the case for the two RpS5 paralogs, of which RpS5b is ovary-switched. Both paralogs shift out of the polysomes to small complexes, corresponding to $40 \mathrm{~S}$ subunits. The patterns exhibited for paralogs were also seen with a canonical RP, RpL40. Together these results indicate that we have identified changes in ribosome protein composition rather than in large non-ribosomal complexes.

\section{Cryo-electron microscopy of testis and ovary ribosomes re- veals a mechanism for inactivation of testis $80 \mathrm{~S}$ ribosomes}

To understand the molecular implications of the paralog switching events we identified by mass spectrometry and western blot, we sought to solve structures of different ribosome populations. Ribosomal complexes were isolated by sucrose gradient centrifugation, in the same way as for the TMT (Figure 1B). Imaging the sample by cryoelectron microscopy (cryo-EM) confirmed that the ribosome complexes were highly pure and concentrated (Supplementary S10A) and a dataset containing $~ 47000$ particles was collected. Three-dimensional classification of this testis $80 \mathrm{~S}$ dataset identified a single structurally distinct class of $80 \mathrm{~S}$ ribosomes, which was refined to an average $3.5 \AA$ resolution (Figure 3A and Supplementary S10B and C). This provided a substantial improvement to the only other D. melanogaster ribosome cryo-EM average at $6 \AA$ resolution, from embryos (59). We performed a similar experiment with ovary $80 \mathrm{~S}$ ribosome preparations, collecting a dataset containing $\sim 200000$ particles, and resulting in an average $3.0 \AA$ resolution (Figure 3B; Supplementary S10D$\mathrm{F}$ ). These averages allowed us to generate atomic models for testis and ovary $80 \mathrm{~S}$ ribosomal complexes (Supplementary Table S2).

Comparison of the testis and ovary $80 \mathrm{~S}$ models revealed that the main difference between them is at the P- and Esites (Figure $3 \mathrm{~A}$ and $\mathrm{B}$ ). While the ovary $80 \mathrm{~S}$ average did not contain any densities in this region, the testis $80 \mathrm{~S}$ average contained densities that did not correspond to a tRNA (Figure 3A, circle). As a comparison, the previously published D. melanogaster average contained densities for an E-tRNA and for elongation factor 2, neither of which are present in our maps (59). By combining information from the testis $80 \mathrm{~S}$ structure and the corresponding TMT data, we identified this density to be the interferon-related developmental regulator 1 (IFRD1) (Figure 3C), which is abundant in the testis $80 \mathrm{~S}$ complexes (54th most abundant protein in testis 80S-TMT1). Of note, rabbit IFRD2, orthologous to $D$. melanogaster IFRD1, was identified in translationally inactive rabbit ribosomes as being bound to $\mathrm{P}$ and E- sites of $\sim 20 \% 80 \mathrm{~S}$ isolated from rabbit reticulocytes (64). Strikingly, in the reticulocytes the presence of IFRD2 is always accompanied by a tRNA in a noncanonical position (termed Z-site). In the testis 80S average no tRNA was found in this region. In mammals (rabbits and humans), IFRD2 is thought to have a role in translational regulation during differentiation (64). Differentiation is a key process during spermatogenesis within the testis, and in this context, it is unsurprising to have found this protein in the testis $80 \mathrm{~S}$. D. melanogaster IFRD1 has considerable amino acid sequence conservation with rabbit IFRD2 (37\% identity, Supplementary S11A and B). The presence of IFRD1 suggests that a significant proportion of the testis $80 \mathrm{~S}$ ribosomes are not actively engaged in translation. The IFRD1 density was not present in the ovary $80 \mathrm{~S}$ structure, suggesting far fewer ribosomes are inactive by this mechanism in the ovary. The presence of IFRD1 does not affect the paralog enrichment events because the testis specific paralog enrichment was identical between the testis $80 \mathrm{~S}$ and testis polysome ribosomes, and polysomes are unlikely to be translational repressed. To verify this, we solved the structure of ribosomes isolated from testis polysomes (cryo-EM average resolution was $4.9 \AA$ ) (Figure 3D and Supplementary S10G-I). It is clear from the density map that IFRD1 is not present in either the P- or E-sites; rather there is density for the E-tRNA in these actively translating ribosomes (Figure $3 \mathrm{C}$ and $\mathrm{D}$ ). In summary, we have solved the structures of testis $80 \mathrm{~S}$, ovary $80 \mathrm{~S}$ and testis polysomal ribosomes, which exhibit differences in translational activity.

\section{Functional implications of paralog-switching events in gonads}

To understand the implications of the enrichment for different RP paralogs between testis and ovary $80 \mathrm{~S}$ ribosomes we probed our two $80 \mathrm{~S}$ cryo-EM structures. By mapping the 12 Drosophila paralog pairs onto our ribosome structures (all paralog pairs except RpS14a and RpS14b, as they have the same amino acid sequences), we identified three clusters in which they localise. (i) Paralogs within the small subunit, including $\mathrm{RpS} 28 \mathrm{a} / \mathrm{b}, \mathrm{RpS} 5 \mathrm{a} / \mathrm{b}$ and $\mathrm{RpS} 19 \mathrm{a} / \mathrm{b}$, map to the head of the $40 \mathrm{~S}$ near the mRNA channel (Figure $4 \mathrm{~A}$ and $\mathrm{B}$ ). (ii) Paralogs within the large subunit tend be surface-exposed. Specifically, RpL22/RpL22-like and RpL24/RpL24-like locate towards the back of the ribosome (Figure 4C-E). (iii) Paralogs that are located in ribosome stalks, RpLP0 and RpL10A, potentially interacting with the mRNA during translation (Figure 4F). Of note, several small subunit paralogs are close to the mRNA channel, pointing towards possible functional differences in mRNA selectivity of the ribosome.

By comparing the atomic models for testis $80 \mathrm{~S}$ and ovary $80 \mathrm{~S}$, switching the paralogs identified by western blot, we found small differences in the paralog positions between ovary and testis ribosomes (Table 1). Specifically, the three 

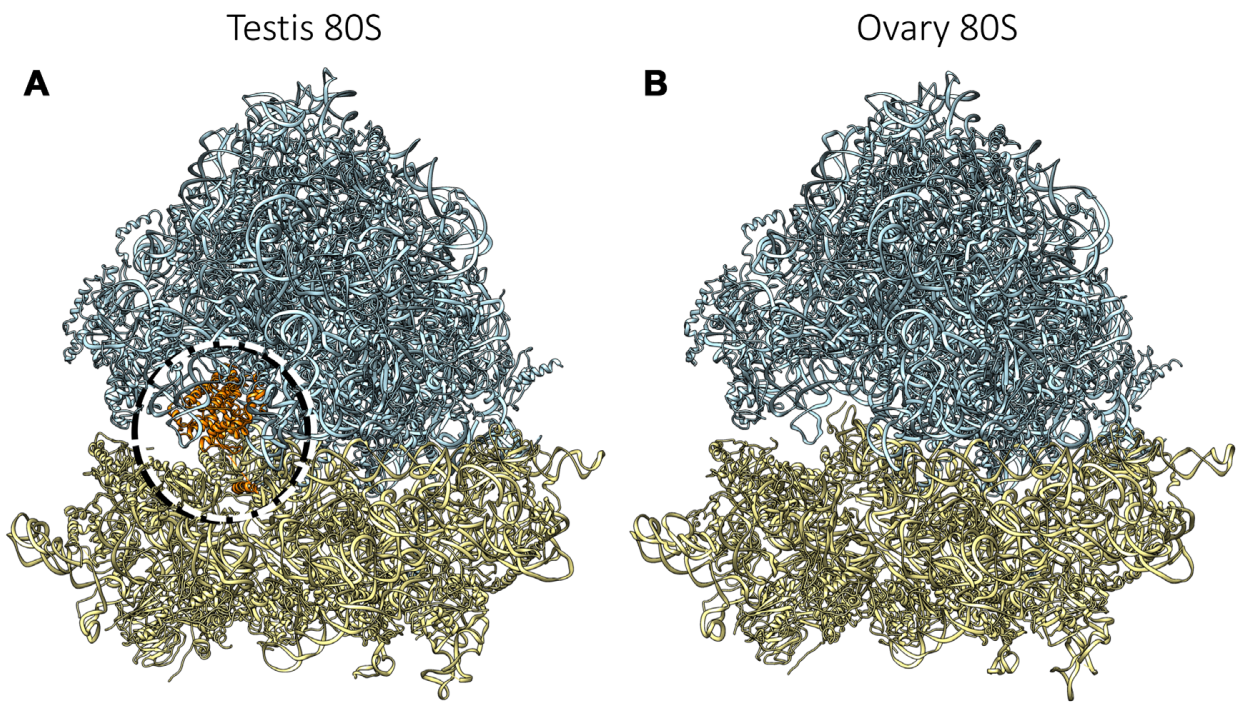

C

D Testis Polysome
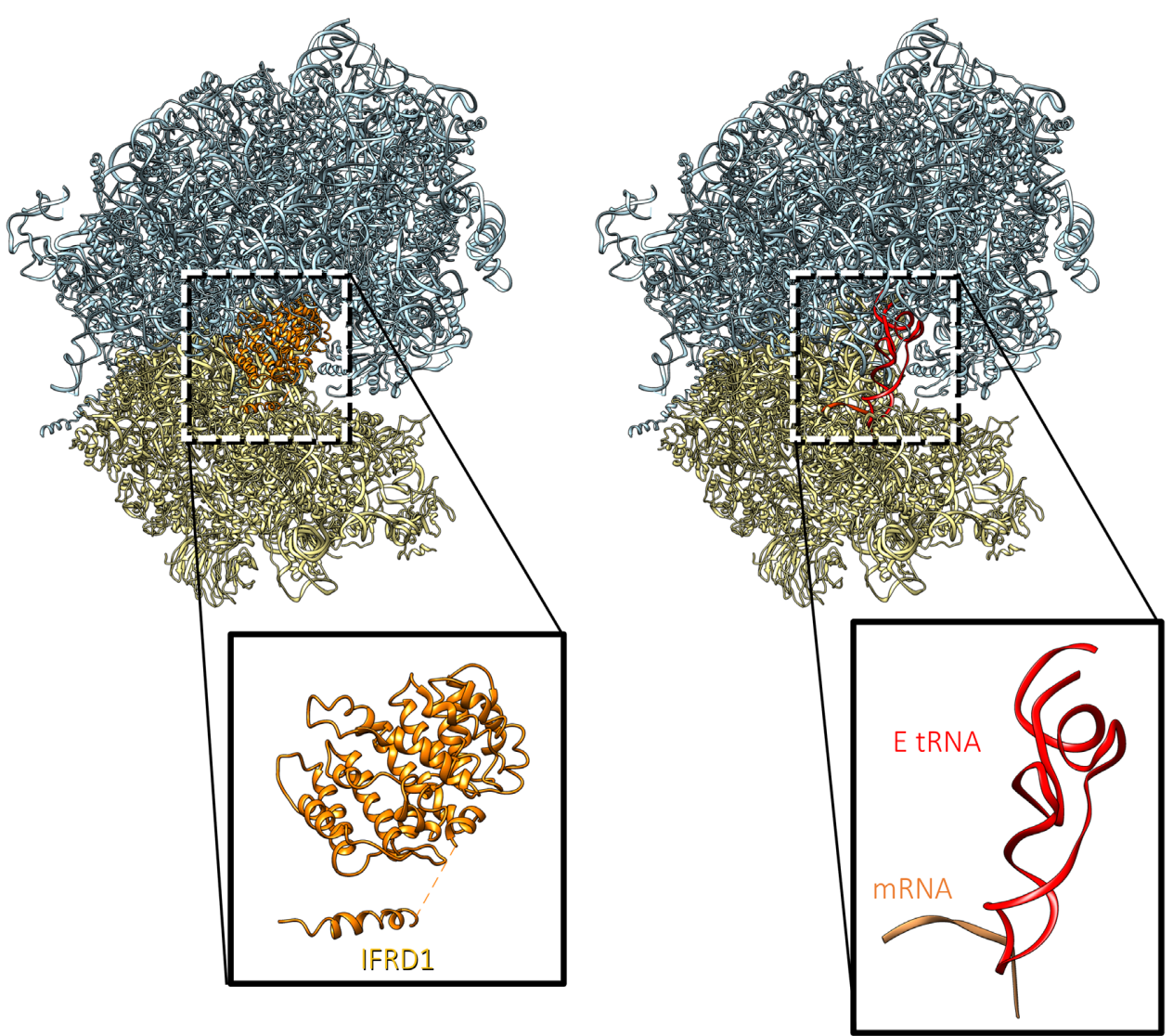

Figure 3. Atomic models of testis and ovary ribosomes. Atomic models of testis $80 \mathrm{~S}$ (A and C), ovary $80 \mathrm{~S}$ (B) and testis polysome (D). Large subunits are light blue and small subunits are yellow. IFRD1 is orange, mRNA is salmon and E tRNA is red. 
A

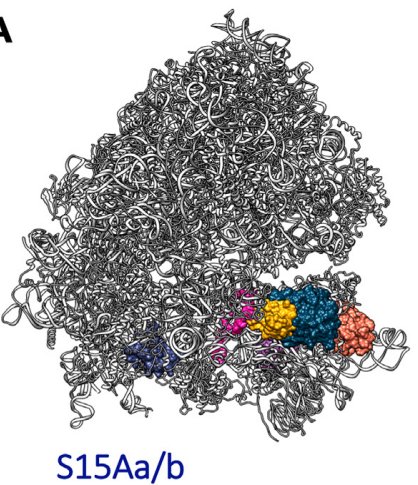

$\mathrm{S} 15 \mathrm{Aa} / \mathrm{b}$

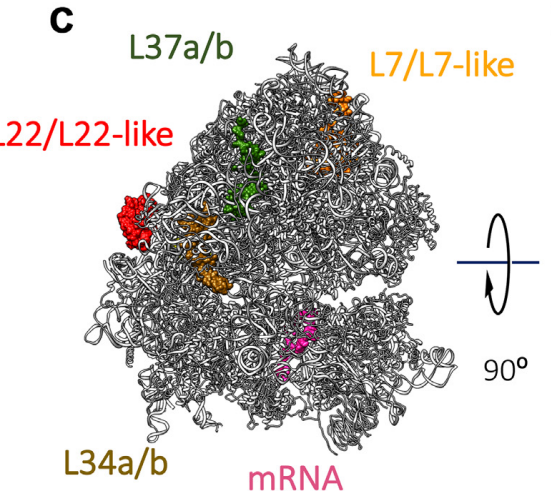

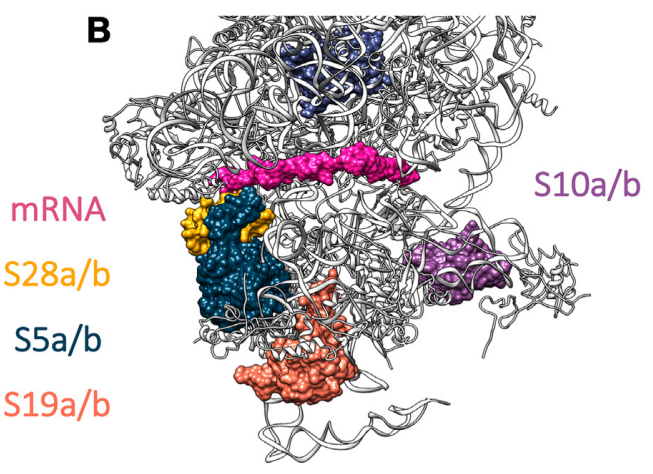

D

Figure 4. Location of D. melanogaster ribosomal paralogs. Ribosomal paralogs mapped to the testis 80S EM average. (A and B) Small subunit paralogs: $\mathrm{RpS15a} / \mathrm{b}$ (medium blue), RpS28a/b (gold), RpS5a/b (navy blue), RpS19a/b (coral) and RpS10a/b (purple); mRNA is pink. Paralogs are shown viewed from the side into mRNA channel (A) and from the top, without the large subunit (B). (C-E) Large subunit paralogs: L37a/b (green), L22/L22-like (red), L7/L7-like (orange), L34a/b (brown) and L24/L24-like (yellow); mRNA is pink. Paralogs are viewed from the side into mRNA channel (C), from the top of the ribosome (D) and from the opposite side of the mRNA channel (E). (F) Paralogs that locate in ribosome stalks: L10Aa/b (dark pink) and LP0/LP0-like (light pink); mRNA is pink. Paralogs are shown viewed from the front of ribosome.

switched paralogs (RpL22-like and RpL37b in testis 80S; and RpS5b in ovary 80S; Figure 2D) showed the largest differences in their atomic models out of all paralogs (Figure 5A-I). Additionally, analysing the fit of both RpL22like and RpL22 into the cryo-EM density of testis $80 \mathrm{~S}$ ribosomes showed a better fit for RpL22-like, further pointing towards a paralog switch (Supplementary S12). This was also the case for RpL37b in testis $80 \mathrm{~S}$ ribosomes (Supplementary S13). Of the paralogs we could not confirm a switch between testis $80 \mathrm{~S}$ and ovary $80 \mathrm{~S}$ by western blot, $\mathrm{RpS19a}, \mathrm{RpS10b}$ and RpS28b showed the largest differences (Supplementary S14 and S15). We note that the differences of the atomic models of these paralogs between testis and ovary $80 \mathrm{~S}$ ribosomes are above the differences for nonparalog models, that were used as control (Table 1). These differences might represent actual paralog switches or could be due to the low resolution at the head of the small subunit. For RpS5a and RpS28b the differences could also be due to their proximity to the E- and P-sites and therefore the position of IFRD1 in the testis 80S (Supplementary S16).

Comparing the amino acid sequences of each paralog pair it is possible to predict that they might contribute different functionality to the ribosome (Table 2 and Supplementary S17). RpL22 and RpL22-like are only 45\% identical, even though they are very similar in length (Figure 6A, Supplementary S17). Unfortunately, the most different region between RpL22 and RpL22-like (i.e., the N-terminal region; Figure 6A), faces the exterior of the ribosome and is not resolved in the cryo-EM density (Figure 6A shows in bold the regions of RpL22 and RpL22-like present in the 
A

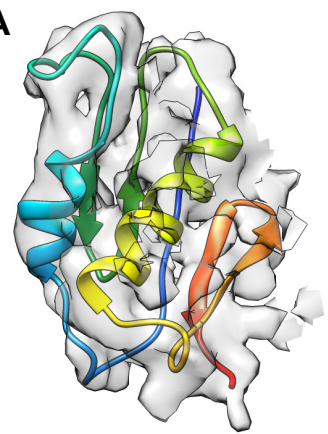

RpL22-like Testis 80S
B

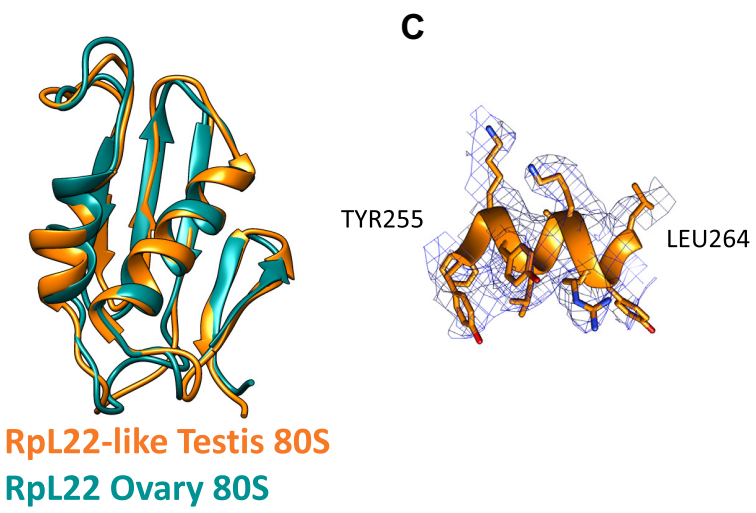

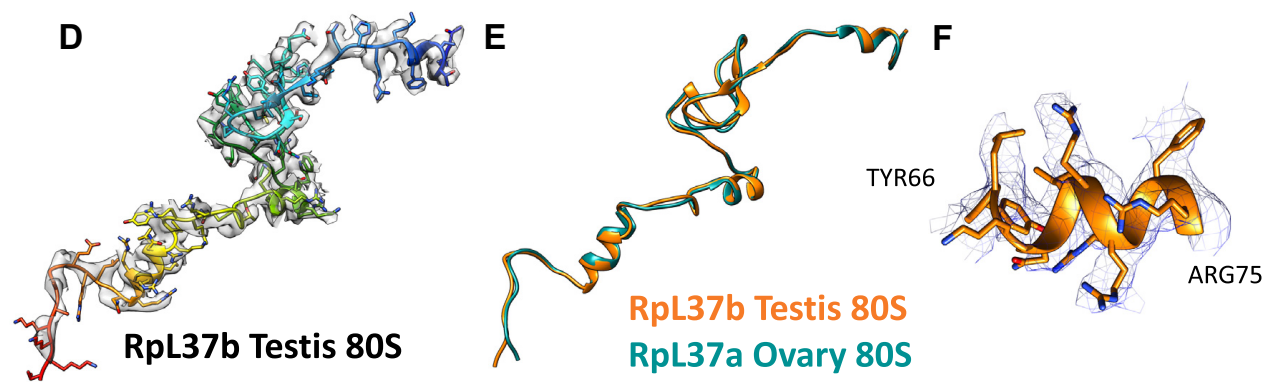

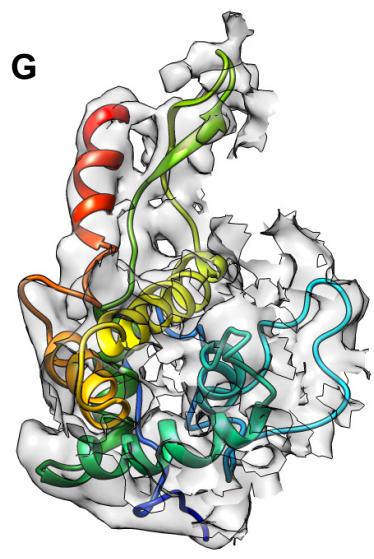

RpS5a Testis 80S
H

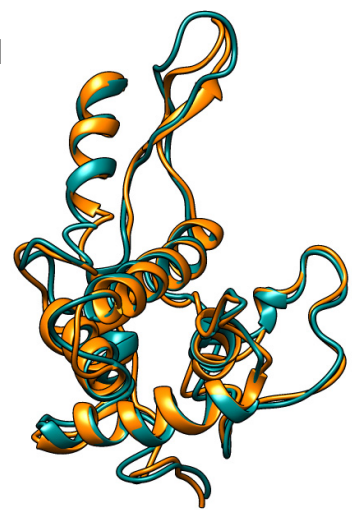

RpS5a Testis 80S

RpS5b Ovary 80S
I

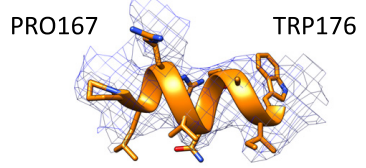

Figure 5. Structural implications of paralog-switching events. Switched paralogs in testis $80 \mathrm{~S}$ vs ovary $80 \mathrm{~S}$ are shown. (A-C) RpL22-like (testis $80 \mathrm{~S})$ and RpL22 (ovary 80S). (D-F) RpL37b (testis 80 S) and RpL37a (ovary 80S). (G-I) RpS5a (testis 80S) and RpS5b (ovary 80S). (A, D and G) Testis 80S atomic model fitted into the EM density. Models are rainbow colored from $\mathrm{N}$-terminus (blue) to C-terminus (red). (B, E and H) Comparison between the testis $80 \mathrm{~S}$ (orange) and the ovary $80 \mathrm{~S}$ (teal) atomic models. (C, F and I) Representative fits of the testis $80 \mathrm{~S}$ atomic models into the EM map.

ovary $80 \mathrm{~S}$ and testis $80 \mathrm{~S}$ reconstructions, respectively). It is possible to imagine that given the majority of these paralogs are localised to the exterior of the ribosome, by switching one for the other might provide a difference exterior surface, with which other associated factors might bind.

\section{Evolution of $R p l 22$ paralogs by independent duplication events}

To probe how widespread paralog switching events might be to facilitate ribosome specialisation we determined the level of conservation of Rpl22 and Rpl22-like in other animal genomes. Orthologs of Rpl22 were identified across a range of animals including Drosophilids. We determined that the paralogous pair Rpl22 and Rpl22-like present in $D$. melanogaster evolved by 3 independent duplication events across the animal clade (Figure 6B). A duplication event unique to the Drosophila clade produced the paralogous pair Rpl22 and Rpl22-like that are identifiable in 6 out of the 12 Drosophila species sampled. The additional 2 duplication events are present in the vertebrate clade and may be the result of whole genome duplication rather than individual gene duplication events. The first of these vertebrate duplications produced the paralog pair RPL22 and RPL22L we observe in humans for example. The second vertebrate RPL22 duplication specific event occurred amongst teleost 
Table 1. Atomic model paralog comparison

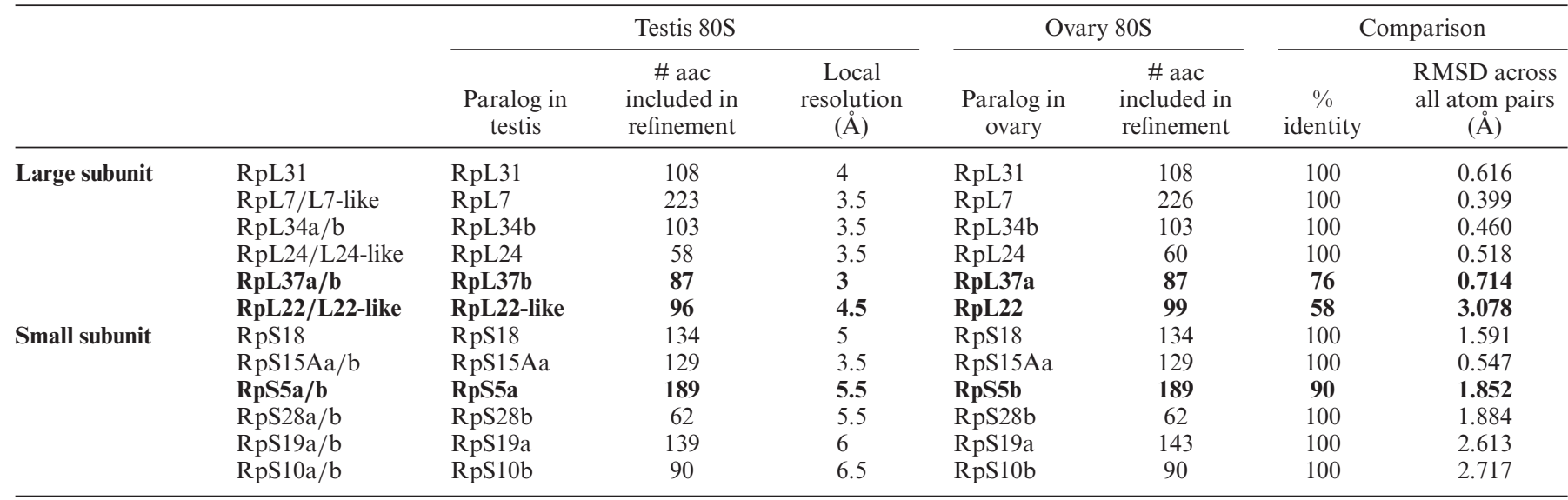

Switched paralogs are highlighted in bold. L31 and S18 do not have paralogs and were used as internal controls. Resolution refers to the local resolution of the testis $80 \mathrm{~S}$ EM average for each paralog. The \% identity refers to identity when comparing the amino acids included in the refinement. RMSD is root-mean-square deviation in A between the atomic models.

Table 2. Summary of paralog pair attributes. For each paralog pair: amino acid length, amino acid identity, tissue enrichment or switching, relationship to human RPs and associated human diseases

\begin{tabular}{|c|c|c|c|c|c|c|c|}
\hline $\begin{array}{l}\text { Canonical } \\
\text { paralog }\end{array}$ & $\begin{array}{l}\text { aa } \\
\text { length }\end{array}$ & $\begin{array}{l}\text { Alternative } \\
\text { paralog }\end{array}$ & $\begin{array}{l}\text { aa } \\
\text { length }\end{array}$ & $\begin{array}{l}\text { Paralog } \\
\text { homology }\end{array}$ & Tissue enrichment & $\begin{array}{l}\text { Human } \\
\text { ortholog(s) }\end{array}$ & Human disease \\
\hline RpL22 & 299 & RpL22-like & 312 & $45 \%$ & Testis RpL22-like & $\begin{array}{l}\text { RpL22 } \\
\text { RpL22L1 }\end{array}$ & Cancer/blood disease. \\
\hline RpS19a & 160 & RpS19b & 159 & $66 \%$ & Testis RpL19b & $\mathrm{RpS} 19$ & Diamond-Blackfan anaemia. Cancer. \\
\hline RpS10a & 163 & RpS10b & 160 & $61 \%$ & Testis RpS10a & $\mathrm{RpS} 10$ & $\begin{array}{l}\text { Diamond-Blackfan anaemia. Aase } \\
\text { syndrome }\end{array}$ \\
\hline RpL7 & 252 & RpL7-like & 257 & $28 \%$ & Ovary 80 S RpL7-like & RpL7 & Cancer. \\
\hline RpLP0 & 317 & RpLP0-like & 256 & $23 \%$ & $\begin{array}{l}\text { Ovary } 80 \mathrm{~S} \\
\text { RpLP0-like }\end{array}$ & RpLP0 & \\
\hline RpS5a & 228 & $\mathrm{RpS} 5 \mathrm{~b}$ & 230 & $76 \%$ & $\begin{array}{l}\text { Ovary }>\text { Embryo }> \\
\text { Testis RpS5b }\end{array}$ & RpS5 & Diamond-Blackfan anaemia. Cancer. \\
\hline
\end{tabular}

fishes and the most parsimonious explanation of pattern of distribution of duplicate copies would suggest subsequent loss in some lineages (Figure 6B). Thus, RPL22 has undergone multiple independent duplication events, generating a complex array of paralogous pairs.

\section{DISCUSSION}

We have characterised the heterogeneity of $80 \mathrm{~S}$ ribosome composition across ribosomes purified from 4 in vivo tissues. The main source of heterogeneity we discovered were paralog-enriching events in the gonads. We have identified 6 testis-enriched paralogs (RpL22-like, RpL37b, RpS19b, $\mathrm{RpS10a}, \mathrm{Rp} 28 \mathrm{a}, \mathrm{RpS15Ab}$ ) and four ovary-enriched paralogs (RpL24-like, RpL7-like, RpL0-like and RpS5b). In addition to being ovary-enriched $\mathrm{RpS} 5 \mathrm{~b}$ is also present in embryo and testis but to a lesser extent. We were able to validate testis-enrichment for RpL22-like, RpL37b and ovaryenrichment for RpS5b by western blots. These westerns also suggest that these alternative paralogs are more abundant than the canonical ones, indicating that paralog-switching is occurring, not simply paralog-enrichment. Furthermore, the enrichment of $\mathrm{RpS} 19 \mathrm{~b}$ and $\mathrm{RpS} 5 \mathrm{~b}$ in the testis is to such an extent that they are at about equal levels to their respective canonical pairs, i.e $\sim 50 \%$ of ribosomes likely contain $\mathrm{RpS} 19 \mathrm{~b}$ and RpS5b in the testis. There are very few differences between the composition of $80 \mathrm{~S}$ and polysome ribosomes across all tissues. Paralog incorporation is not simply the consequence of transcriptional regulation of these paralogous genes. Rather there is modulation at the level of the translation of some of these proteins and/or incorporation into the ribosome. We can be sure that the difference we have detected are within ribosomes, rather than other large RNPs, as EDTA treatment disrupts our complexes.

We have solved the cryo-EM structures of three different ribosome complexes purified from complex in vivo tissues; $80 \mathrm{~S}$ ribosomes from the testis $(3.5 \AA), 80 \mathrm{~S}$ ribosomes from the ovary $(3.0 \AA)$ and polysomal ribosomes from the testis (4.9 $\AA$ ), improving the resolution from the only other previous ribosome from $D$. melanogaster (59). One key difference was that the testis $80 \mathrm{~S}$ structure contains IFRD1, which is a homolog of the human IFRD2. Its presence indicates there is functional homology between the two proteins in inhibiting mRNA translation through the ribosome, during differ- 


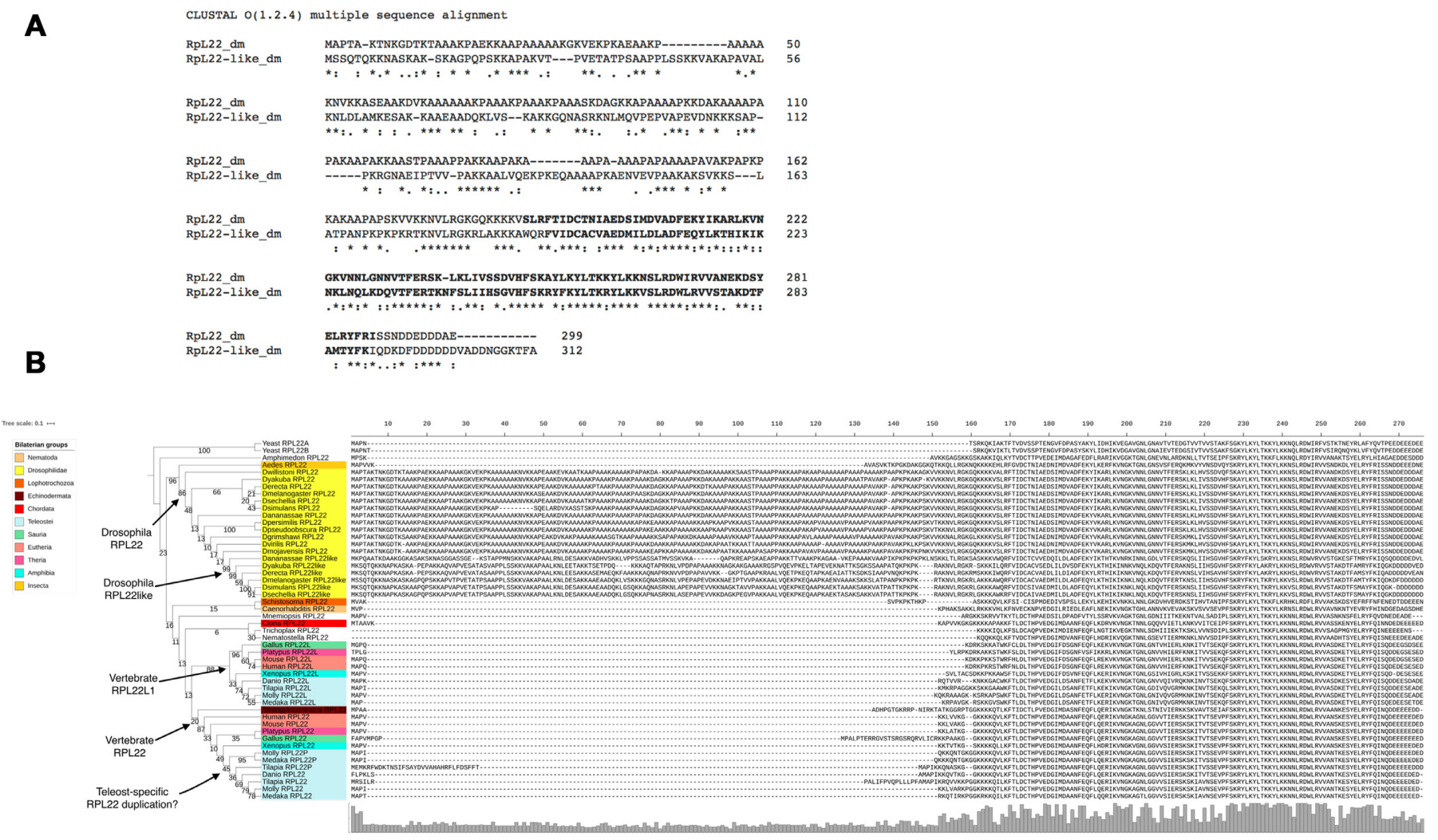

Figure 6. Evolution of RpL22 paralogs by independent duplication events. (A) Alignment of RpL22 and RpL22-like amino acid sequences for the two $D$. melanogaster proteins. In bold are amino acids included in the atomic models. (B) Phylogenetic tree for RpL22 and RpL22-like paralogs across a range of animal genomes. The three duplication events detected are indicated.

entiation. In mammals IFRD2 was seen in differentiating reticulocytes (64), whilst in our work we found IFRD1 in the testis $80 \mathrm{~S}$ (but not in the ovary $80 \mathrm{~S}$ ), where it could be involved in regulation of translation during the differentiation of spermatozoa, which is central to the function of the testis.

D. melanogaster paralogs are localised in three clusters: (a) the head of the 40S near the mRNA channel, (b) the surface-exposed back of the large subunit and c) ribosome stalks, potentially interacting with the mRNA during translation. The position of these three clusters provides potential explanations of how specialisation might be achieved, mechanistically. Differences in amino acid sequence and precise position of the testis and ovary switched paralogs (Figure 5) could potentially affect the interaction of the mRNA and the ribosome, specifically during initiation when $40 \mathrm{~S}$ ribosomes are recruited to the $5^{\prime}$ end of mRNAs. The back of the $60 \mathrm{~S}$ would provide an ideal site for additional protein factors to differentially bind to ribosomes containing these RPs, with the potential to regulate ribosome activity. This is particularly true for the RpL22 and RpL22-like paralog pair, which has the lowest sequence identity between each other, $45 \%$. The termini of these proteins are likely to be dynamic given the lack of density for them in our EM maps. Our phylogenomic analysis suggests that the modulation of this part of the exterior ribosome surface is in common across many organisms, and that the generation of paralogs has occurred independently three times for $R p L 22$. Therefore, this provides a potential mechanism for ribosome regulation across many eukaryotes. Although paralogs are not conserved across a range of organisms, and many are limited to Drosophilids, there are many organisms with many RP paralog pairs, including human (19 pairs) and Arabidopsis (80 pairs). Therefore, these potential mechanisms of ribosome regulation could be conserved, if not the precise details.

mRNA translational regulation is important in the testis and ovary. For example, many testis-specific translation components exist to enable tight regulation such as eIF4E-3 (29). The result we find here, that the gonads are important sites of ribosome heterogeneity, suggests that RP paralogenriching, and potentially paralog-switching, might also play a part in this regulation via ribosome specialisation. The paralog-enrichment events in the testis we have identified involve pairs where the 'canonical' paralog in the pair is located on the $\mathrm{X}$ chromosome. One hypothesis for the expression of the 'alternative' paralog in testis enables gene dosage to be maintained during meiotic sex chromosome inactivation, which occurs in males (65). However, additional functions could have evolved as well as this.

The importance of the paralog-switching event between $\mathrm{RpS} 5 \mathrm{a}$ and $\mathrm{RpS} 5 \mathrm{~b}$ has recently been functionally characterised in the Drosophila ovary (34). Females without $\mathrm{RpS} 5 \mathrm{~b}$ produce ovaries with developmental and fertility defects, whilst those without RpS5a have no defects. RpS5b specifically binds to mRNAs encoding proteins with func- 
tions enriched for mitochondrial and metabolic GO terms in the ovary, suggesting ovary RpS5b containing ribosomes translate this specific pool of mRNAs (34). It will be interesting to see how widespread this finding is for $\mathrm{RpS} 5 \mathrm{~b}$ in other tissues, since this is a frequently enriched paralog; it is enriched in embryo and testis to a lesser extent. It has been known for some time that mutations in RpS5a produce a Minute phenotype (including infertility), so it seems likely that these two paralogs each have biologically important roles in the fly. RpS5a and RpS5b have also been seen to exhibit tissue-specific expression in $A$. thaliana, in a developmentally regulated manner (15). atRpS5a was suggested to be more important than atRpS5b during differentiation, because of its expression pattern, but the regulation mechanism remains elusive in $A$. thaliana.

The function of the RpL22 and RpL22-like paralog pair in the Drosophila testis has been previously explored and it has been suggested that the two proteins are not functionally redundant in development or spermatogenesis $(66,67)$. Further work is needed to directly link effects on ribosome composition and mRNA translational output, as the two paralogs interact with different pools of mRNA in the testis (67).

Interestingly, we found little differences between $80 \mathrm{~S}$ and polysomal ribosome composition, apart from an enrichment of RpL24-like in 80S ribosomes in the testis and head. RpL7-like was also enriched in testis $80 \mathrm{~S}$ and RpL38 in the ovary polysome. Eukaryotic orthologs of RpL24-like are thought to have a role in the formation and processing pre-60S complexes, with RpL24 replacing RpL24-like at the very end of processing (68). Given that we saw enrichment of RpL24-like in 80S compared to polysomes in the testis and the head, this suggests that a proportion of these $80 \mathrm{~S}$ complexes could represent the final stage of testing $80 \mathrm{~S}$ competency. It is not clear why this would be the case in only these tissues. Rpl24-like is present in other insects and some non-insect arthropods (FlyBase). A paralogswitching event between RpL24 and RpL24-like could be important in translation initiation or indeed provide a platform for additional proteins to bind to the ribosome, given $\mathrm{RpL} 24 / \mathrm{RpL} 24-$ like is located close to $\mathrm{RpL} 22 / \mathrm{RpL} 22$-like.

Several of the RPs that have gonad-specific paralog pairs (including RpS19, RpS5, RpS10, RpS28 and RpL22 $(69,70))$ have been linked with human diseases, specifically Diamond-Blackfan anemia and cancer (Table 2). Thus, it will be important to uncover if they contribute to mRNA translation regulation and work in vivo using Drosophila could help understand how they contribute to the translation of specific mRNAs.

One of the few canonical RPs we found to be differentially incorporated was $\mathrm{RpS} 11$ in the head $80 \mathrm{~S}$ ribosomes. In humans RPS11 phosphorylation is linked to Parkinson's disease (71) and higher levels of RPS11 correlate with poorer prognosis in glioblastoma patients (72). Therefore, understanding RpS11 levels in Drosophila head could provide a mechanism of future exploration for dissecting the molecular mechanisms by which RP mutations result in human disease.

Altogether our data reveal ribosome heterogeneity occurs in a tissue specific manner through differential incorporation of ribosomal paralog proteins. We further show para- $\log$ switching events in the gonads and our structural analysis has provided insights into how these switches might regulate translation mechanistically. Additionally, our evolutionary data suggest heterogeneity may represent a conserved mechanism of translation regulation across eukaryotes.

\section{DATA AVAILABILITY}

The mass spectrometry proteomics data have been deposited to the ProteomeXchange Consortium via PRIDE partner repository with the dataset identified PDX026227. The EM-density maps for testis $80 \mathrm{~S}$, testis polysomes and ovary $80 \mathrm{~S}$ are deposited in the EMDB under the accession numbers EMD-10622, EMD-10623 and EMD-10624. The refined models are deposited in the PDB under accession codes 6XU6, 6XU7and 6XU8.

\section{SUPPLEMENTARY DATA}

Supplementary Data are available at NAR Online.

\section{ACKNOWLEDGEMENTS}

Mass spectrometry was performed by Bristol University Proteomics Facility and we would like to thank Kate Heesom for her assistance. We thank the Astbury Biostructure Laboratory (ABSL) Facility staff for assisting with cryoEM data collection. Electron microscopy image processing was partially undertaken on ARC3, part of the High Performance Computing (HPC) facilities at the University of Leeds. We also thank Laura Wilkinson Hewitt and Brian Jackson of University of Leeds Protein Production Facility (PPF) for RpL22 and RpL22-like antibody purification. CGPM and MJOC would like to thank The University of Nottingham for HPC facilities and the School of Life Sciences for research support. RpS5a and RpS5b antibodies were kindly gifted by Jian Kong and Paul Lasko (34). We would also like to thank Pavel Baranov and Gary Loughran for insightful discussions.

\section{FUNDING}

J.A. and J.F. are funded by the University of Leeds (University Academic Fellow scheme); this work was funded by Royal Society [RSG $\backslash$ R $1 \backslash 180102]$; BBSRC [BB/S007407/1]; Wellcome Trust ISSF [105615/Z/14/Z]; White Rose University Consortium - Collaborative Grant and MRC [MR/N000471/1]; M.A. was funded from BBSRC DTP [BB/M011151/1]; all Electron Microscopy was performed at ABSL, which was funded by the University of Leeds and the Wellcome Trust [108466/Z/15/Z]. Funding for open access charge: University of Leeds. Conflict of interest statement. None declared.

\section{REFERENCES}

1. Kondrashov,N., Pusic,A., Stumpf,C.R., Shimizu,K., Hsieh,A.C., Xue,S., Ishijima,J., Shiroishi,T. and Barna,M. (2011)

Ribosome-mediated specificity in Hox mRNA translation and vertebrate tissue patterning. Cell, 145, 383-397. 
2. Shi,Z., Fujii,K., Kovary,K.M., Genuth,N.R., Röst,H.L., Teruel,M.N. and Barna,M. (2017) Heterogeneous ribosomes preferentially translate distinct subpools of mRNAs Genome-wide. Mol. Cell, 67, 71-83.

3. Segev,N. and Gerst,J.E. (2018) Specialized ribosomes and specific ribosomal protein paralogs control translation of mitochondrial proteins. J. Cell Biol., 217, 117-126.

4. García-Marcos,A., Sánchez,S.A., Parada,P., Eid,J., Jameson,D.M., Remacha,M., Gratton,E. and Ballesta,J.P. (2008) Yeast ribosomal stalk heterogeneity in vivo shown by two-photon FCS and molecular brightness analysis. Biophys. J., 94, 2884-2890.

5. Krogh,N., Jansson,M.D., Häfner,S.J., Tehler,D., Birkedal,U., Christensen-Dalsgaard,M., Lund,A.H. and Nielsen,H. (2016) Profiling of 2'-O-Me in human rRNA reveals a subset of fractionally modified positions and provides evidence for ribosome heterogeneity. Nucleic Acids Res., 44, 7884-7895.

6. Simsek,D., Tiu,G.C., Flynn,R.A., Byeon,G.W., Leppek,K., Xu,A.F., Chang,H.Y. and Barna,M. (2017) The mammalian ribo-interactome reveals ribosome functional diversity and heterogeneity. Cell, 169, 1051-1065.

7. Kearse,M.G., Chen,A.S. and Ware,V.C. (2011) Expression of ribosomal protein L22e family members in Drosophila melanogaster: rpL22-like is differentially expressed and alternatively spliced. Nucleic Acids Res., 39, 2701-2716.

8. Carroll,A.J., Heazlewood,J.L., Ito,J. and Millar,A.H. (2008) Analysis of the Arabidopsis cytosolic ribosome proteome provides detailed insights into its components and their post-translational modification. Mol. Cell. Proteomics, 7, 347-369.

9. Natchiar,S.K., Myasnikov,A.G., Kratzat,H., Hazemann,I. and Klaholz,B.P. (2017) Visualization of chemical modifications in the human 80 S ribosome structure. Nature, 551, 472-477.

10. Norris,K., Hopes,T. and Aspden,J.L. (2021) Ribosome heterogeneity and specialization in development. Wiley Interdiscip. Rev. RNA, 12, e1644.

11. Dinman,J.D. (2016) Pathways to specialized ribosomes: the brussels lecture. J. Mol. Biol., 428, 2186-2194.

12. Bortoluzzi,S., Alessi,F., Romualdi,C. and Danieli,G.A. (2001) Differential expression of genes coding for ribosomal proteins in different human tissues. Bioinformatics, 17, 1152-1157.

13. Guimaraes,J.C. and Zavolan,M. (2016) Patterns of ribosomal protein expression specify normal and malignant human cells. Genome Biol., $17,236$.

14. Szakonyi,D. and Byrne,M.E. (2011) Ribosomal protein L27a is required for growth and patterning in Arabidopsis thaliana. Plant $J$., 65, 269-281.

15. Weijers,D., Franke-van Dijk,M., Vencken,R.J., Quint,A., Hooykaas,P. and Offringa,R. (2001) An Arabidopsis Minute-like phenotype caused by a semi-dominant mutation in a RIBOSOMAL PROTEIN S5 gene. Development, 128, 4289-4299.

16. Marygold,S.J., Coelho,C.M. and Leevers,S.J. (2005) Genetic analysis of RpL38 and RpL5, two minute genes located in the centric heterochromatin of chromosome 2 of Drosophila melanogaster. Genetics, 169, 683-695.

17. Martinez Barrio,A., Eriksson,O., Badhai,J., Fröjmark,A.S., Bongcam-Rudloff,E., Dahl,N. and Schuster,J. (2009) Targeted resequencing and analysis of the Diamond-Blackfan anemia disease locus RPS19. PLoS One, 4, e6172.

18. Ludwig,L.S., Gazda,H.T., Eng,J.C., Eichhorn,S.W., Thiru,P., Ghazvinian,R., George,T.I., Gotlib,J.R., Beggs,A.H., Sieff,C.A. et al. (2014) Altered translation of GATA1 in Diamond-Blackfan anemia. Nat. Med., 20, 748-753.

19. Thurmond,J., Goodman,J.L., Strelets,V.B., Attrill,H., Gramates,L.S., Marygold,S.J., Matthews,B.B., Millburn,G., Antonazzo,G., Trovisco, V. et al. (2019) FlyBase 2.0: the next generation. Nucleic Acids Res., 47, D759-D765.

20. Barakat,A., Szick-Miranda,K., Chang,I.F., Guyot,R., Blanc,G., Cooke,R., Delseny,M. and Bailey-Serres,J. (2001) The organization of cytoplasmic ribosomal protein genes in the Arabidopsis genome. Plant Physiol., 127, 398-415.

21. Sugihara,Y., Honda,H., Iida,T., Morinaga,T., Hino,S., Okajima,T., Matsuda,T. and Nadano,D. (2010) Proteomic analysis of rodent ribosomes revealed heterogeneity including ribosomal proteins L10-like, L22-like 1, and L39-like. J. Proteome Res., 9, 1351-1366.
22. Williams,M.E. and Sussex,I.M. (1995) Developmental regulation of ribosomal protein L16 genes in Arabidopsis thaliana. Plant J., 8, 65-76.

23. Richter,J.D. and Lasko,P. (2011) Translational control in oocyte development. Cold Spring Harb. Perspect. Biol., 3, a002758.

24. Qin,X., Ahn,S., Speed,T.P. and Rubin,G.M. (2007) Global analyses of mRNA translational control during early Drosophila embryogenesis. Genome Biol., 8, R63.

25. Boria,I., Garelli,E., Gazda,H.T., Aspesi,A., Quarello,P., Pavesi,E., Ferrante,D., Meerpohl,J.J., Kartal,M., Da Costa,L. et al. (2010) The ribosomal basis of Diamond-Blackfan Anemia: mutation and database update. Hum. Mutat., 31, 1269-1279.

26. Slaidina,M. and Lehmann,R. (2014) Translational control in germline stem cell development. J. Cell Biol., 207, 13-21.

27. Yu,J., Lan,X., Chen,X., Yu,C., Xu,Y., Liu,Y., Xu,L., Fan,H.Y. and Tong,C. (2016) Protein synthesis and degradation are essential to regulate germline stem cell homeostasis in Drosophila testes. Development, 143, 2930-2945.

28. Vedelek,V., Bodai,L., Grézal,G., Kovács,B., Boros,I.M., Laurinyecz,B. and Sinka,R. (2018) Analysis of Drosophila melanogaster testis transcriptome. BMC Genomics, 19, 697.

29. Ghosh,S. and Lasko,P. (2015) Loss-of-function analysis reveals distinct requirements of the translation initiation factors eIF4E, eIF4E-3, eIF4G and eIF4G2 in Drosophila spermatogenesis. PLoS One, 10, e0122519.

30. Lambertsson,A. (1998) The minute genes in Drosophila and their molecular functions. Adv. Genet., 38, 69-134.

31. Kongsuwan,K., Yu,Q., Vincent,A., Frisardi,M.C., Rosbash,M., Lengyel,J.A. and Merriam,J. (1985) A Drosophila minute gene encodes a ribosomal protein. Nature, 317, 555-558.

32. Bass,T.M., Grandison,R.C., Wong,R., Martinez,P., Partridge,L. and Piper,M.D. (2007) Optimization of dietary restriction protocols in Drosophila. J. Gerontol. A Biol. Sci. Med. Sci., 62, 1071-1081.

33. Consortium, U. (2019) UniProt: a worldwide hub of protein knowledge. Nucleic Acids Res., 47, D506-D515.

34. Kong,J., Han,H., Bergalet,J., Bouvrette,L.P.B., Hernández,G., Moon,N.S., Vali,H., Lécuyer,É. and Lasko,P. (2019) A ribosomal protein S5 isoform is essential for oogenesis and interacts with distinct RNAs in Drosophila melanogaster. Sci. Rep., 9, 13779.

35. Lyne,R., Smith,R., Rutherford,K., Wakeling,M., Varley,A., Guillier,F., Janssens,H., Ji,W., Mclaren,P., North,P. et al. (2007) FlyMine: an integrated database for Drosophila and Anopheles genomics. Genome Biol., 8, R129.

36. Brown,J.B., Boley,N., Eisman,R., May,G.E., Stoiber,M.H., Duff,M.O., Booth,B.W., Wen,J., Park,S., Suzuki,A.M. et al. (2014) Diversity and dynamics of the Drosophila transcriptome. Nature, 512, 393-399.

37. Graveley,B.R., Brooks,A.N., Carlson,J.W., Duff,M.O., Landolin,J.M., Yang,L., Artieri,C.G., van Baren,M.J., Boley,N., Booth,B.W. et al. (2011) The developmental transcriptome of Drosophila melanogaster. Nature, 471, 473-479.

38. Thompson,R.F., Iadanza,M.G., Hesketh,E.L., Rawson,S. and Ranson,N.A. (2019) Collection, pre-processing and on-the-fly analysis of data for high-resolution, single-particle cryo-electron microscopy. Nat. Protoc., 14, 100-118.

39. Fernandez-Leiro,R. and Scheres,S.H.W. (2017) A pipeline approach to single-particle processing in RELION. Acta Crystallogr. D Struct. Biol., 73, 496-502.

40. Zheng,S.Q., Palovcak,E., Armache,J.P., Verba,K.A., Cheng,Y. and Agard,D.A. (2017) MotionCor2: anisotropic correction of beam-induced motion for improved cryo-electron microscopy. Nat. Methods, 14, 331-332.

41. Zhang,K. (2016) Gctf: Real-time CTF determination and correction. J. Struct. Biol., 193, 1-12.

42. Zivanov,J., Nakane,T., Forsberg,B.O., Kimanius,D., Hagen,W.J., Lindahl,E. and Scheres,S.H. (2018) New tools for automated high-resolution cryo-EM structure determination in RELION-3. Elife, 7, e42166.

43. Scheres,S.H. and Chen,S. (2012) Prevention of overfitting in cryo-EM structure determination. Nat. Methods, 9, 853-854.

44. Nakane,T., Kimanius,D., Lindahl,E. and Scheres,S.H. (2018) Characterisation of molecular motions in cryo-EM single-particle data by multi-body refinement in RELION. Elife, 7, e36861. 
45. Pettersen,E.F., Goddard,T.D., Huang,C.C., Couch,G.S., Greenblatt,D.M., Meng,E.C. and Ferrin,T.E. (2004) UCSF chimera - a visualization system for exploratory research and analysis. J. Comput. Chem., 25, 1605-1612.

46. Waterhouse,A., Bertoni,M., Bienert,S., Studer,G., Tauriello,G., Gumienny,R., Heer,F.T., de Beer,T.A.P., Rempfer,C., Bordoli,L. et al. (2018) SWISS-MODEL: homology modelling of protein structures and complexes. Nucleic Acids Res., 46, W296-W303.

47. Afonine,P.V., Klaholz,B.P., Moriarty,N.W., Poon,B.K., Sobolev,O.V., Terwilliger,T.C., Adams,P.D. and Urzhumtsev,A. (2018) New tools for the analysis and validation of cryo-EM maps and atomic models. Acta Crystallogr. D Struct. Biol., 74, 814-840.

48. Emsley,P., Lohkamp,B., Scott,W.G. and Cowtan,K. (2010) Features and development of Coot. Acta Crystallogr. D. Biol. Crystallogr., 66, 486-501.

49. Cunningham,F., Achuthan,P., Akanni,W., Allen,J., Amode,M.R., Armean,I.M., Bennett,R., Bhai,J., Billis,K., Boddu,S. et al. (2019) Ensembl 2019. Nucleic Acids Res., 47, D745-D751.

50. Altschul,S.F., Gish,W., Miller,W., Myers,E.W. and Lipman,D.J. (1990) Basic local alignment search tool. J. Mol. Biol., 215, 403-410.

51. Edgar,R.C. (2004) MUSCLE: multiple sequence alignment with high accuracy and high throughput. Nucleic Acids Res., 32, 1792-1797.

52. Katoh,K. and Standley,D.M. (2013) MAFFT multiple sequence alignment software version 7: improvements in performance and usability. Mol. Biol. Evol., 30, 772-780.

53. Loytynoja,A. (2014) Phylogeny-aware alignment with PRANK. Methods Mol. Biol., 1079, 155-170.

54. Blackburne,B.P. and Whelan,S. (2012) Measuring the distance between multiple sequence alignments. Bioinformatics, 28, 495-502.

55. Thompson,J.D., Plewniak,F., Ripp,R., Thierry,J.C. and Poch,O. (2001) Towards a reliable objective function for multiple sequence alignments. J. Mol. Biol., 314, 937-951.

56. Capella-Gutierrez,S., Silla-Martinez,J.M. and Gabaldon,T. (2009) trimAl: a tool for automated alignment trimming in large-scale phylogenetic analyses. Bioinformatics, 25, 1972-1973.

57. Nguyen,L.T., Schmidt,H.A., Haeseler,A. and Minh,B.Q. (2015) IQ-TREE: a fast and effective stochastic algorithm for estimating maximum-likelihood phylogenies. Mol. Biol. Evol., 32, 268-274.

58. Kalyaanamoorthy,S., Minh,B.Q., Wong,T.K.F., von Haeseler,A. and Jermiin,L.S. (2017) ModelFinder: fast model selection for accurate phylogenetic estimates. Nat. Methods, 14, 587-589.

59. Anger,A.M., Armache,J.P., Berninghausen,O., Habeck,M., Subklewe,M., Wilson,D.N. and Beckmann,R. (2013) Structures of the human and Drosophila 80 S ribosome. Nature, 497, 80-85.

60. Thomas,A., Lee,P.J., Dalton,J.E., Nomie,K.J., Stoica,L., Costa-Mattioli,M., Chang,P., Nuzhdin,S., Arbeitman,M.N. and
Dierick,H.A. (2012) A versatile method for cell-specific profiling of translated mRNAs in Drosophila. PLoS One, 7, e40276.

61. Aspden,J.L., Eyre-Walker,Y.C., Phillips,R.J., Amin,U., Mumtaz,M.A., Brocard,M. and Couso,J.P. (2014) Extensive translation of small open reading frames revealed by Poly-Ribo-Seq. Elife, 3, e03528.

62. Heyer,E.E. and Moore,M.J. (2016) Redefining the translational status of 80 S monosomes. Cell, 164, 757-769.

63. Branco-Price,C., Kawaguchi,R., Ferreira,R.B. and Bailey-Serres,J. (2005) Genome-wide analysis of transcript abundance and translation in Arabidopsis seedlings subjected to oxygen deprivation. Ann Bot, 96, 647-660

64. Brown,A., Baird,M.R., Yip,M.C., Murray,J. and Shao,S. (2018) Structures of translationally inactive mammalian ribosomes. Elife, 7, e40486.

65. Jiang,L., Li,T., Zhang,X., Zhang,B., Yu,C., Li,Y., Fan,S., Jiang,X., Khan,T., Hao,Q. et al. (2017) RPL10L is required for male meiotic division by compensating for RPL10 during meiotic sex chromosome inactivation in mice. Curr. Biol., 27, 1498-1505.

66. Mageeney,C.M., Kearse,M.G., Gershman,B.W., Pritchard,C.E., Colquhoun,J.M. and Ware,V.C. (2018) Functional interplay between ribosomal protein paralogues in the eRpL22 family in Drosophila melanogaster. Fly (Austin), 12, 143-163.

67. Mageeney,C.M. and Ware,V.C. (2019) Specialized eRpL22 paralogue-specific ribosomes regulate specific mRNA translation in spermatogenesis in. Mol. Biol. Cell, 30, 2240-2253.

68. Saveanu,C., Namane,A., Gleizes,P.E., Lebreton,A., Rousselle,J.C., Noaillac-Depeyre,J., Gas,N., Jacquier,A. and Fromont-Racine,M (2003) Sequential protein association with nascent 60S ribosomal particles. Mol. Cell. Biol., 23, 4449-4460.

69. Campagnoli,M.F., Ramenghi,U., Armiraglio,M., Quarello,P., Garelli,E., Carando,A., Avondo,F., Pavesi,E., Fribourg,S., Gleizes,P.E. et al. (2008) RPS19 mutations in patients with Diamond-Blackfan anemia. Hum. Mutat., 29, 911-920.

70. Cao,B., Fang,Z., Liao,P., Zhou,X., Xiong,J., Zeng,S. and Lu,H. (2017) Cancer-mutated ribosome protein L22 (RPL22/eL22) suppresses cancer cell survival by blocking p53-MDM2 circuit. Oncotarget, 8, 90651-90661.

71. Martin,I., Dawson,V.L. and Dawson,T.M. (2011) Recent advances in the genetics of Parkinson's disease. Annu. Rev. Genomics Hum. Genet., 12, 301-325.

72. Yong,W.H., Shabihkhani,M., Telesca,D., Yang,S., Tso,J.L., Menjivar,J.C., Wei,B., Lucey,G.M., Mareninov,S., Chen,Z. et al. (2015) Ribosomal proteins RPS11 and RPS20, two Stress-Response markers of glioblastoma stem cells, are novel predictors of poor prognosis in glioblastoma patients. PLoS One, 10, e0141334. 